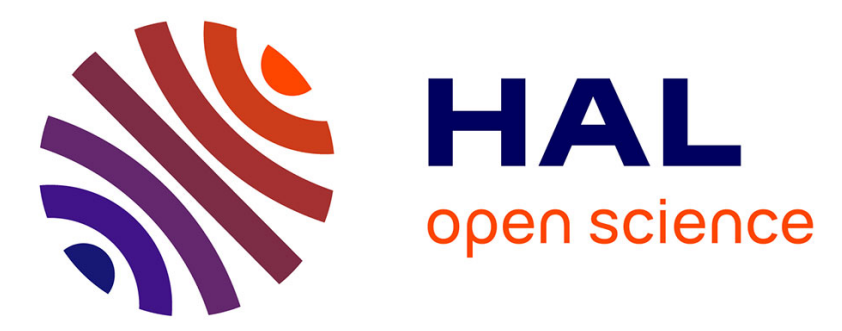

\title{
Accounting for Polydispersion in the Eulerian Large Eddy Simulation of the Two-Phase Flow in an Aeronautical-type Burner
}

\author{
Aymeric Vié, Stéphane Jay, Bénédicte Cuenot, Marc Massot
}

\section{To cite this version:}

Aymeric Vié, Stéphane Jay, Bénédicte Cuenot, Marc Massot. Accounting for Polydispersion in the Eulerian Large Eddy Simulation of the Two-Phase Flow in an Aeronautical-type Burner. Flow, Turbulence and Combustion, 2013, 90 (3), pp.545-581. 10.1007/s10494-012-9440-x . hal-00650605v2

\section{HAL Id: hal-00650605 \\ https://hal.science/hal-00650605v2}

Submitted on 29 Dec 2011

HAL is a multi-disciplinary open access archive for the deposit and dissemination of scientific research documents, whether they are published or not. The documents may come from teaching and research institutions in France or abroad, or from public or private research centers.
L'archive ouverte pluridisciplinaire HAL, est destinée au dépôt et à la diffusion de documents scientifiques de niveau recherche, publiés ou non, émanant des établissements d'enseignement et de recherche français ou étrangers, des laboratoires publics ou privés. 


\title{
Accounting for Polydispersion in the Eulerian Large Eddy Simulation of the Two-Phase Flow in an Aeronautical-type Burner
}

\author{
Aymeric Vié · Stéphane Jay · \\ Bénédicte Cuenot • Marc Massot
}

Received: date / Accepted: date

\begin{abstract}
A major issue for the simulation of two-phase flows in engines concerns the modeling of the liquid disperse phase, either in the Lagrangian or the Eulerian approach. In the perspective of massively parallel computing, the Eulerian approach seems to be more suitable, as it uses the same algorithm as the gaseous phase solver. However taking into account the whole physics of a turbulent spray, especially in terms of polydispersity, requires an additional modeling effort. The Mesoscopic Eulerian Formalism (MEF) [13] accounts for the effect of turbulence on the dispersed phase, and was extended to the Large Eddy Simulation framework [42], but is limited to monodisperse flows. In [39], the influence of polydispersity on resolved and unresolved turbulent motions of the disperse phase was highlighted, and a first model was proposed, based on size-conditioned statistics. Starting from this idea, a coupling between the MEF and the Multifluid Approach (MA) [31] is proposed. The MA decomposes the Eulerian phase into several fluids classes called sections, and corresponding to size intervals. Each section uses then size-conditioned closures. The original idea of this work is to use the MEF closures independently in each section, taking into account the mean droplet size of
\end{abstract}

A.Vié · S. Jay

IFP énergies nouvelles, 1 et 4 avenue de Bois Préau, F92852, Rueil-Malmaison Cedex, France Tel. (A.V.): +33-(0)1-41 131854

Fax: +33-(0)1-47 028035

E-mail: aymeric.vie@em2c.ecp.fr, stéphane.jay@ifpen.fr

Present address: EM2C, CNRS et Ecole Centrale Paris, F92295, Chatenay-Malabry, France

B. Cuenot

CERFACS, 42 avenue Gaspard Coriolis, F31057, Toulouse Cedex 01, France

Tel.: +33-(0)5-61 193044

Fax: +33-(0)5-61 193000

E-mail: benedicte.cuenot@cerfacs.fr

M. Massot

EM2C, CNRS et Ecole Centrale Paris, F92295, Chatenay-Malabry, France

Tel. : +33-(0)1-41 131081

Fax: +33-(0)1-47 028035

Center for Turbulence Research, Stanford University, California 94305-4035, USA

E-mail: marc.massot@em2c.ecp.fr 
this section. This new approach, called Multifluid Mesoscopic Eulerian Formalism (MMEF), is then able to capture polydispersion with associated size-conditioned turbulent dynamics. First, the importance of polydispersity and the ability of MMEF to capture it are highlighted with a $0 \mathrm{D}$ evaporation case and a $2 \mathrm{D}$ vortex case, showing its impact on dynamics in both size and physical spaces. Then, the MMEF is applied to the MERCATO configuration of ONERA [18]. Results are compared to monodisperse Eulerian, Lagrangian and experimental results.

Keywords Large Eddy Simulation, Two-phase flow, Polydisperse sprays, Eulerian Mesoscopic, Multifluid, Aeronautical burner

\section{Introduction}

Considering available computational resources, Large Eddy Simulation (LES) becomes more and more attractive for industrial applications. For single-phase reacting flows, LES has already shown its great potential for aeronautical combustors $[34,55]$ or automotive engines [30].

For two-phase reacting flows, simulating the whole physics from injection to turbulent dispersion is still a challenge as the simulation of the primary atomization requires accurate models and high computational resources [41, 9, 19, 47]. Furthermore most methods used to simulate the primary atomization are not adapted to subsequent turbulent spray. Fortunately, the primary atomization occurs in a limited zone near the injector and, in first approximation, can be accounted for by representative boundary conditions $[37,54]$.

Assuming point-particles by comparison to the smallest flow resolved scales (estimated by the mesh size), the disperse phase statistics can be described by the number density function (NDF), which is solution of the Williams-Boltzmann Equation (WBE, [64]), describing its evolution in phase space. Solving the WBE directly is completely unreachable, due to the high number of dimensions of the phase space. Instead Lagrangian methods, like the Direct Simulation Monte Carlo [3] method, build statistics by tracking stochastic particles, or like the Discrete Particle Simulation (DPS, [50]), consist in tracking particles of one individual realization of the disperse phase. Numerous studies have used this approach with success $[1,44,23]$, and shown its great potential (ease of modeling, computational time with small number of particles), but also its main drawbacks (projection on the Eulerian grid to couple with the gas phase, computational time with high number of particles, statistical convergence). Finally, Eulerian approaches solve the moments of the NDF (such as mean number density or volume fraction). This implies a loss of information, as moments are integrals of the NDF, but results in Eulerian equations to which classical methods for the gas phase may be directly applied. The CPU cost then depends only on the mesh size. In such methods, two main issues arise, concerning the modeling of the effect of turbulence on the spray, and the polydispersion in size of the spray. Those two issues are of primary importance for industrial applications, as they are responsible for the fuel mass fraction distribution in the combustion chamber, controlling ignition and combustion in the full device.

Turbulent spray dispersion models in the Eulerian formulation depend on the Stokes number range. For small Stokes number $(\mathrm{St}<<1)$, equilibrium approaches 
can be used [12], which only consider the diffusive effect of turbulence on the zeroth order moment for low Stokes numbers. For moderate Stokes number (St $\approx 1$ ), the disperse phase dynamics become more complex: strong segregation effects occur and the disperse phase motion exhibits correlated and uncorrelated components [57, 49]. To describe such physics, Février et al. [13] have developed the Mesoscopic Eulerian Formalism (MEF), which considers one droplet size only, but introduces a velocity dispersion around the mean velocity. Kaufmann et al. [27] and Moreau et al. [42] addressed the problem of accurate closures for the uncorrelated stresses imposed by the velocity dispersion in the LES context. The resulting model was applied to automotive engine injection [35], aeronautical jets in crossflow [22], or aeronautical configurations such as the one considered here $[52,53]$. More recently, Zaichik et al. [66] proposed a LES formalism based on the work of Reeks [48] and Zaichik et al. [67], taking into account the effect of subgrid turbulence directly at the kinetic level. This approach is still to be applied in more complex configurations.

Taking into account size polydispersion adds another complexity to Eulerian models. A first method consists in sampling the NDF by a set of monodisperse Eulerian phases [31], as is done in a Lagrangian stochastic approach. This method is relatively simple when Eulerian phases do not interact, i.e. when no collision occur. However this approach suffers from intrinsic poor statistical convergence, since only a very limited number of samples can be used. Another method is based on the quadrature approximation $[33,16,65]$ of the moments. Gaussian quadratures are usually used, leading to simple closures for the evolution in real and phase space. Although good results have been obtained for coalescing sprays [15], even with turbulence [2], Fox et al. [15] demonstrate the difficulties of such methods to reproduce evaporation for continuous distributions, as they cannot recover point wise values of the NDF. Today's most promising formalism is the Multifluid Approach (MA, [31]). Dividing the size space in sections [20], this formalism reproduces the evaporation of polydisperse sprays, and captures primary aspects of the size/velocity correlations in complex flows. Validation of this formalism was done through $0 \mathrm{D}$ and $1 \mathrm{D}$ simple test cases with one or two size moments per section $[32,10]$, showing the robust mathematical basis.

Previous work on a real configuration using both the Lagrangian approach [56] and the MEF $[29,51,53]$ has shown that the size polydispersion does not greatly influence the mean velocity, but has significant effects on fluctuations and on the distribution of kerosene vapor. A good agreement was obtained between Lagrangian monodisperse and MEF results, showing the importance and good modeling of velocity dispersion. Introducing polydispersity in Lagrangian simulation however resulted in a significant deviation from monodisperse results.

Starting from these conclusions, the goal of this work is to introduce the size polydispersion in the Eulerian framework, by coupling MEF and MA approaches, to take into account the size dependent effect of turbulence on velocity dispersion of the spray dynamics, through size-conditioned closures. This new formalism is called the Multifluid Mesoscopic Eulerian Formalism (MMEF), and is implemented in the AVBP code to simulate the MERCATO configuration [18].

This paper is organized as follows. First the MMEF is introduced. Emphasis is made on the differences between strategies to solve the WBE. In section 3, the aeronautical configuration MERCATO is presented with the associated operating point and numerical methods. In section 4 , a $0 \mathrm{D}$ evaporation case and a $2 \mathrm{D}$ vortex 
case are proposed to analyze the importance of polydispersity in the MERCATO conditions on similar reduced configurations. Finally, results obtained with the Lagrangian approach, the MEF and the MMEF are compared to experiment, with regards to mean and RMS velocities, as well as size-conditioned statistics.

\section{Modeling approach}

The modeling approach considered here aims at the description of a spray, i.e. a dispersed liquid phase resulting from the disintegration of a liquid core, and is restricted by the following assumptions:

- droplets are spherical and smaller than Kolmogorov length scale,

- the spray is dilute,

- the liquid-gas density ratio is high.

These assumptions lead to a point-particle description without collisions and with drag force as the only external force acting on droplets (effect of gravity is neglected).

In this framework, the goal is to predict the NDF $f_{p}$ of the droplets, driven by the Williams-Boltzmann Equation (WBE):

$$
\frac{\partial f_{p}}{\partial t}+\frac{\partial c_{p, j} f_{p}}{\partial x_{j}}+\frac{1}{m_{p}} \frac{\partial F_{p, j} f_{p}}{\partial c_{p, j}}+\frac{\partial E_{S} f_{p}}{\partial S_{p}}+\frac{\partial E_{T} f_{p}}{\partial T_{p}}=0
$$

where $t, x_{j}, c_{p, j}, m_{p}, S_{p}$, and $T_{p}$ are respectively the time, position, velocity, mass, surface and temperature of the droplet. The quantities $F_{p, j}, E_{S}, E_{T}$ are respectively the drag force and the mass and energy transfer due to evaporation:

$$
\begin{aligned}
F_{p, j} & =\frac{c_{p, j}-u_{g} @ p, j}{\tau_{p}} \\
E_{S} & =\frac{4 \sqrt{\pi}}{\rho_{l} S_{p}^{3 / 2}} \dot{m}_{p} \\
E_{T} & =\frac{\phi_{p}^{c}}{m_{p} C_{p, l}} .
\end{aligned}
$$

where $u_{g} @ p, j, \tau_{p}, \dot{m}_{p}$ and $\phi_{p}^{c}$ are respectively the gas velocity at droplet position, the droplet relaxation time, the mass rate due to evaporation, and the heat transfer rate due to conduction. The evaporation model is derived for an isolated spherical droplet at the thermodynamic equilibrium with a surrounding quiescent mixture of ideal gas. Within the assumption of infinitely fast thermal conductivity in the liquid, the evaporating rate $\dot{m}_{p}$ and the heat transfer rate $\phi_{p}^{c}$ are [58, 28]:

$$
\begin{aligned}
\dot{m}_{p} & =-\sqrt{\pi S_{p}} \operatorname{Sh}\left[\rho D_{f}\right] \ln \left(1+\mathrm{B}_{\mathrm{M}}\right) \\
\phi_{p}^{c} & =-\phi_{g}^{c}+\dot{m}_{p} L_{v}\left(T_{p}\right)=\sqrt{\pi S_{p}} \mathrm{Nu} \lambda\left(T_{\infty}-T_{p}\right) \frac{1+\mathrm{B}_{\mathrm{T}}}{\mathrm{B}_{\mathrm{T}}}+\dot{m}_{p} L_{v}\left(T_{p}\right)
\end{aligned}
$$

where $L_{v}\left(T_{p}\right)$ is the latent heat of vaporization at temperature $T_{p}, \mathrm{~B}_{\mathrm{M}}$ and $\mathrm{B}_{\mathrm{T}}$ stand for the Spalding numbers of mass and temperature respectively, accounting for the gas mixture modification in the gas layer surrouding the droplet, following 
the $1 / 3^{r d}-2 / 3^{r d}$ rule [21]. The Nusselt $\mathrm{Nu}$ and Sherwood Sh numbers are modified following [14] to account for the effect of non-vanishing slip velocity between droplet and carrier phase.

In the LES context, the Favre $\left({ }^{\wedge}\right)$ and Reynolds $\left(^{-}\right)$filtering procedures are introduced:

$$
\begin{aligned}
\bar{m}_{\Phi} \widehat{f_{\Phi}}(x) & =\int_{\Delta_{f}} m_{\Phi} f_{\Phi}(x) G_{\Delta_{f}}\left(x-x^{\prime}\right) \mathrm{d} x^{\prime} \\
\overline{f_{\Phi}}(x) & =\int_{\Delta_{f}} f_{\Phi}(x) G_{\Delta_{f}}\left(x-x^{\prime}\right) \mathrm{d} x^{\prime}
\end{aligned}
$$

\subsection{Lagrangian approach}

A first way to solve Eq. 1 is to build statistics from a sample of individual particles. The Lagrangian equations associated to Eq. 1 are [7]:

$$
\begin{aligned}
\frac{\mathrm{d} x_{i}}{\mathrm{~d} t} & =c_{p, i} \\
\frac{\mathrm{d} c_{p, i}}{\mathrm{~d} t} & =F_{p, i} \\
\frac{\mathrm{d} S_{p}}{\mathrm{~d} t} & =E_{S} \\
\frac{\mathrm{d} T_{p}}{\mathrm{~d} t} & =E_{T}
\end{aligned}
$$

LES filtering of Eqs. 9-12 leads to unclosed terms, due to the non-linear dependence of the right hand side on the gas phase. These terms can be accounted for like in $[44,46]$, but they are often neglected as in $[1,56]$, so that all the source terms are written from filtered quantities. This type of simulation solves only one realisation of the disperse phase, and thus suffers from a slow statistical convergence. To overcome this problem, Eulerian methods can be envisaged, as they solve the NDF directly through its moments.

\subsection{Mesoscopic Eulerian Formalism}

The MEF developed by Février et al. [13] is designed to solve turbulent two phase flows with one-way coupling, i.e. the gas phase is not influenced by the disperse phase. This formalism is an extension of the Bi-fluid model [11], and accounts for the effect of turbulence on the disperse phase by solving the Random Uncorrelated Motion (RUM), which plays a similar role than pressure and viscosity in gas dynamics. More details about the derivation and the main assumptions are available in $[13]$. 
Instead of solving for Eq. 1 on the $\mathrm{NDF} f_{p}$, one can solve for the moments, by integrating $f_{p}$ over velocities, size and temperature:

$$
\begin{aligned}
n_{l} & =\int f_{p} d c_{p} d S_{p} \mathrm{~d} T_{p} \\
m_{l} & =\int m_{p} f_{p} d c_{p} d S_{p} \mathrm{~d} T_{p} \\
u_{l, i} & =\frac{1}{n_{l}} \int c_{p, i} f_{p} d c_{p} d S_{p} \mathrm{~d} T_{p} \\
\delta \Theta_{l} & =\frac{1}{2 n_{l}} \int\left(c_{p, i}-U_{l, i}\right)^{2} f_{p} d c_{p} d S_{p} \mathrm{~d} T_{p} \\
H_{l} & =\frac{1}{n_{l}} \int C_{p, l} T_{p} f_{p} d c_{p} d S_{p} \mathrm{~d} T_{p}
\end{aligned}
$$

where $n_{l}, m_{l}, U_{l, i}, \delta \Theta_{l}$, and $H_{l}$ are respectively the number density, mass, velocity, Random Uncorrelated Energy (RUE), and enthalpy of the liquid phase at position $x$ and time $t$. The RUE is the key-point of MEF, as it allows to capture velocity dispersion of the spray for moderate Stokes number, and represents most of the modeling effort. The MEF is monodisperse, i.e. there is only one droplet surface $S_{l}$ at a position $x$ and a time $t$ :

$$
S_{l}(x, t)=\left(\frac{m_{l}}{n_{l}} \frac{6 \sqrt{\pi}}{\rho_{l}}\right)^{2 / 3}
$$

Introducing the moments in the WBE and applying the LES filter to the resulting equations lead to LES filtered equations [42]:

$$
\begin{aligned}
& \frac{\partial}{\partial t} \bar{n}_{l}+\frac{\partial}{\partial x_{m}} \bar{n}_{l} \widehat{u}_{l, m}=0 \\
& \frac{\partial}{\partial t} \bar{m}_{l}+\frac{\partial}{\partial x_{m}} \bar{m}_{l} \widehat{u}_{l, m}=\bar{E}(1) \\
& \frac{\partial}{\partial t} \bar{m}_{l} \widehat{u}_{l, i}+\frac{\partial}{\partial x_{m}} \bar{m}_{l} \widehat{u}_{l, i} \widehat{u}_{l, m}=\bar{E}\left(\widehat{u}_{l, i}\right)+\bar{F}_{d}-\frac{\partial}{\partial x_{m}} \bar{m}_{l}\left(\widehat{\delta R}_{l, i m}+\tau_{l, i m}\right) \\
& \frac{\partial}{\partial t} \bar{m}_{l} \delta \widehat{\Theta}_{l}+\frac{\partial}{\partial x_{m}} \bar{m}_{l} \delta \widehat{\Theta}_{l} \widehat{u}_{l, m}=\bar{E}\left(\delta \widehat{\Theta}_{l}\right)+\bar{W}_{d}+\bar{\Pi}+\Pi_{S G S}-\frac{1}{2} \frac{\partial}{\partial x_{m}} \bar{m}_{l}\left(\widehat{\delta S}_{l, i i m}\right) \\
& \frac{\partial}{\partial t} \bar{m}_{l} \widehat{H}_{l}+\frac{\partial}{\partial x_{m}} \bar{m}_{l} \widehat{H}_{l} \widehat{u}_{l, m}=\bar{E}\left(\widehat{H}_{l}\right)+\widehat{\phi}_{l}
\end{aligned}
$$

where $E(\Phi)=\dot{m}_{l} \Phi$ are the evaporation source terms, $F_{d}$ is the drag force, $\delta R_{l}$ is the uncorrelated stress tensor, $\tau_{l}$ is the subgrid stress tensor, $\delta S_{l}$ is the RUE diffusion tensor, $W_{d}$ is the RUE source term due to drag force, $\Pi$ and $\Pi_{S G S}$ are the RUE production due to resolved and subgrid motions, and $\phi_{l}$ is the heat transfer. 
2.2.1 Closures

The drag force $F_{d}$ and its RUM contribution $W_{d}$ are:

$$
\begin{aligned}
\bar{F}_{d} & =-\bar{m}_{l} \frac{\widehat{u}_{l, i}-\widehat{u}_{g, i}}{\tau_{p}} \\
\bar{W}_{d} & =-2 \frac{\bar{m}_{l}}{\tau_{p}} \delta \widehat{\Theta}_{l}
\end{aligned}
$$

where the relaxation time $\tau_{p}$ is determined by the droplet surface $S_{l}$. The second order tensor $\widehat{\delta R}_{l, i m}$ is decomposed into isotropic and deviatoric parts:

$$
\widehat{\delta R}_{l, i j}=\frac{\widehat{\delta R}_{l, i i}}{3} \delta_{i j}+\widehat{\delta R}_{l, i j}^{*}=P_{R U M} \delta_{i j}+\widehat{\delta R}_{l, i j}^{*}
$$

where $P_{R U M}=2 / 3 \widehat{\delta \Theta}_{l}$. The deviatoric part is closed using a viscous assumption [27]:

$$
\widehat{\delta R}_{l, i j}^{*}=-\nu_{R U M}\left(\frac{\partial \widehat{u}_{l, i}}{\partial x_{j}}+\frac{\partial \widehat{u}_{l, j}}{\partial x_{i}}-\frac{2}{3} \frac{\partial \widehat{u}_{l, k}}{\partial x_{k}} \delta_{i j}\right)
$$

where $\nu_{R U M}=\tau_{p} \widehat{\delta \Theta}_{l} / 3$. The third order tensor $\widehat{\delta S}_{l, i i j}$ is closed using gradient diffusion law:

$$
\widehat{\delta S}_{p, i i j}=-\kappa_{R U M} \frac{\partial \widehat{\delta \Theta}_{l}}{\partial x_{j}}
$$

where $\kappa_{R U M}=10 / 27 \tau_{p} \delta \widehat{\Theta}_{l}$. Finally, the RUE production $\bar{\Pi}$ is modeled as:

$$
\bar{\Pi}=-\bar{m}_{l} \widehat{\delta R}_{l, i m} \frac{\partial \widehat{u}_{l, i}}{\partial x_{m}}
$$

Following [42], the subgrid tensor $T_{l, i j}$ is closed using a Smagorinsky model for the deviatoric part and a Yoshizawa model for the isotropic part:

$$
\tau_{l, i j}=-C_{s}^{2} \Delta_{f}^{2}\left|\hat{S}_{l}\right|\left(\hat{S}_{l, i j}-\frac{\delta_{i j}}{3} \hat{S}_{l, k k}\right)+C_{l}^{2} \Delta_{f}^{2}\left|\hat{S}_{l}\right|^{2} \delta_{i j}
$$

where $\widehat{S}_{l, i j}=\frac{\partial \widehat{u}_{l, i}}{\partial x_{j}}+\frac{\partial \widehat{u}_{l, j}}{\partial x_{i}}, C_{l}=0.116$ and $C_{s}=0.16$ are constants, and $\Delta_{f}$ is the grid size. Subgrid diffusion term is neglected in Eq. 22, whereas the subgrid production term considers that the subgrid scale motion is totally transfered to the RUM:

$$
\Pi_{S G S}=-\bar{m}_{l} T_{l, i m} \frac{\partial \widehat{u}_{l, i}}{\partial x_{m}}
$$

The evaporation rate $\dot{m}_{l}$ and heat transfer $\phi_{l}$ are calculated using the droplet diameter, and taking the gas properties at infinity as the local gas properties:

$$
\begin{aligned}
\dot{m}_{l} & =-\sqrt{\pi S_{l}} \operatorname{Sh}\left[\rho D_{f}\right] \ln \left(1+\mathrm{B}_{\mathrm{M}}\right) \\
\phi_{l} & =\sqrt{\pi S_{l}} \mathrm{Nu} \lambda\left(T_{g}-T_{l}\right) \frac{1+\mathrm{B}_{\mathrm{T}}}{\mathrm{B}_{\mathrm{T}}}+\dot{m}_{l} L_{v}\left(T_{l}\right)
\end{aligned}
$$

where $T_{l}=H_{l} / m_{l} C_{p, l}$ is the liquid phase temperature. Filtered evaporation terms are calculated as $\bar{E}(\widehat{\Phi})=\dot{m}_{l} \widehat{\Phi}$. 
2.3 Multifluid Mesoscopic Eulerian Formalism

To extend the MEF to polydisperse sprays, it is proposed to couple the MEF to the MA of Laurent et al. [31]. The derivation of this new formalism called Multifluid Mesoscopic Eulerian Formalism (MMEF) is further detailed in [61] and is presented in the following.

The main idea is to divide the size space in $N$ intervals $\Omega_{k}=\left[S_{p}^{(k)}, S_{p}^{(k+1)}\right]$ of fixed length, called sections [20]. Each section is calculated with its own set of equations, and interacts with the others sections through droplet size changes only, for example due to evaporation. Moments are then now defined for each section $k$ :

$$
\begin{aligned}
n_{l}^{(k)} & =\int_{\Omega_{k}} f_{p} d c_{p} d S_{p} \mathrm{~d} T_{p} \\
m_{l}^{(k)} & =\int_{\Omega_{k}} m_{p} f_{p} d c_{p} d S_{p} \mathrm{~d} T_{p} \\
u_{l, i}^{(k)} & =\frac{1}{m_{l}^{(k)}} \int_{\Omega_{k}} m_{p} c_{p, i} f_{p} d c_{p} d S_{p} \mathrm{~d} T_{p} \\
\delta \Theta_{l}^{(k)} & =\frac{1}{2 m_{l}^{(k)}} \int_{\Omega_{k}} m_{p}\left(c_{p, i}-U_{l, i}^{(k)}\right)\left(c_{p, i}-U_{l, i}^{(k)}\right) f_{p} d c_{p} d S_{p} \mathrm{~d} T_{p} \\
H_{l}^{(k)} & =\frac{1}{m_{l}^{(k)}} \int_{\Omega_{k}} m_{p} C_{p, l} T_{p} f_{p} d c_{p} d S_{p} \mathrm{~d} T_{p}
\end{aligned}
$$

where superscript . ${ }^{(k)}$ qualifies quantities integrated over the section $\left[S_{p}^{(k)}, S_{p}^{(k+1)}\right]$. The resulting LES filtered equations for the MMEF are similar to MEF equations:

$$
\begin{aligned}
& \frac{\partial}{\partial t} \bar{m}_{l}^{(k)}+\frac{\partial}{\partial x_{m}} \bar{m}_{l}^{(k)} \widehat{u}_{l, m}^{(k)}= \widehat{E}^{(k)}(1) \\
& \frac{\partial}{\partial t} \bar{m}_{l}^{(k)} \widehat{u}_{l, i}^{(k)}+\frac{\partial}{\partial x_{m}} \bar{m}_{l}^{(k)} \widehat{u}_{l, i}^{(k)} \widehat{u}_{l, m}^{(k)}= \widehat{E}^{(k)}\left(u_{l, i}^{(k)}\right)+F_{d, i}^{(k)} \\
&-\frac{\partial}{\partial x_{m}}\left(\bar{m}_{l}^{(k)} \widehat{\delta R}_{l, i m}^{(k)}+\tau_{l, i m}^{(k)}\right) \\
& \frac{\partial}{\partial t} \bar{m}_{l}^{(k)} \widehat{\delta \Theta}_{l}^{(k)}+\frac{\partial}{\partial x_{m}} \bar{m}_{l}^{(k)} \widehat{\delta \Theta}_{l}^{(k)} \widehat{u}_{l, m}^{(k)}= \widehat{E}^{(k)}\left(\delta \Theta_{l}^{(k)}\right)+W_{d}^{(k)}+\bar{\Pi}^{(k)}+\Pi_{S G S}^{(k)} \\
&-\frac{1}{2} \frac{\partial}{\partial x_{m}} \bar{m}_{l}^{(k)}\left(\widehat{\delta S}_{l, i i m}^{(k)}\right) \\
& \frac{\partial}{\partial t} \bar{m}_{l}^{(k)} \widehat{H}_{l}^{(k)}+\frac{\partial}{\partial x_{m}} \bar{m}_{l}^{(k)} \widehat{H}_{l}^{(k)} \widehat{u}_{l, m}^{(k)}=\widehat{E}^{(k)}\left(H_{l}^{(k)}\right)+\widehat{\phi}_{l}^{(k)}
\end{aligned}
$$

with the same definitions for source terms as in 2.2 , but restricted to each section $k$.

\subsubsection{Closures}

All closures are written as for the MEF approach, applying the definitions to the sectional moments quantities. Only the evaporation terms take a different form, as they imply sectional interactions. These terms are closed using the first order multifluid approach [31]:

$$
\bar{E}^{(k)}\left(\Phi^{(k)}\right)=E_{1}^{(k+1)} \bar{m}_{l}^{(k+1)} \Phi^{(k+1)}-E_{1}^{(k)} \bar{m}_{l}^{(k)} \Phi^{(k)}-E_{2}^{(k)} \bar{m}_{l}^{(k)} \Phi^{(k)}
$$


where $E_{1}^{(k)}=2 E_{S}^{(k)} S^{(k)} /\left(S^{(k+1)^{2}}-S^{(k)^{2}}\right)$ corresponds to the droplet flux from section $k$ to section $k-1$ due to surface decrease and $E_{2}^{(k)}=3 E_{S}^{(k)} /\left(S^{(k+1)}+S^{(k)}\right)$ corresponds to the droplet flux from section $k$ to the gas phase due to droplet mass loss, and where $E_{S}^{(k)}$ is the mass transfer rate defined in Eq. 1. For the RUE equation, an additional term is needed to account for the modification of the mean velocity induced by the evaporative fluxes:

$$
\begin{aligned}
\bar{E}^{(k)}\left(\widehat{\delta \Theta}_{l}^{(k)}\right)= & E_{1}^{(k+1)} \bar{m}_{l}^{(k+1)} \widehat{\delta \Theta}_{l}^{(k+1)}-E_{1}^{(k)} \bar{m}_{l}^{(k)} \widehat{\delta \Theta}_{l}^{(k)}-E_{2}^{(k)} \bar{m}_{l}^{(k)} \widehat{\delta \Theta}_{l}^{(k)} \\
& +E_{1}^{(k+1)} \bar{m}_{l}^{(k+1)} \frac{\left(\widehat{u}_{l, i}^{(k+1)}-\widehat{u}_{l, i}^{(k)}\right)^{2}}{2}
\end{aligned}
$$

The MA accounts for the effect of polydispersion on evaporation and drag force. The MMEF goes further in the modeling, because it considers the effect of polydispersion on the turbulent motion by the resolved scales through the sizeconditioned random uncorrelated energy. As this motion is directly related to the Stokes number, a more precise description of the overall dynamics is expected.

\subsection{Equations for the gas phase}

Knowing now the coupling terms between the liquid and gas phases, the LES equations of the gas phase are introduced. Indeed, all models considered here are in a two-way coupling framework in terms of drag and evaporation. As stated in [13], a rigorous derivation of the moment equations would have to consider the effect of two-way coupling at the kinetic level. However, in the case of small Stokes number relatively to the smallest resolved scales, Février et al. [13] suggest that MEF stays valid for a two-way coupling. Gas phase equations are then similar to pure gaseous flows, with additional source terms resulting from exchanges with the liquid:

$$
\begin{aligned}
\frac{\partial}{\partial t} \bar{\rho}_{g}+\frac{\partial}{\partial x_{m}} \bar{\rho}_{g} \widehat{u}_{g, m} & =\Phi_{\rho_{g}} \\
\frac{\partial}{\partial t} \bar{\rho}_{g} \widehat{u}_{g, i}+\frac{\partial}{\partial x_{m}} \bar{\rho}_{g} \widehat{u}_{g, i} \widehat{u}_{g, m} & =\Phi_{\rho_{g} u_{g, i}}-\frac{\partial \widehat{P}}{\partial x_{i}}+\frac{\partial}{\partial x_{m}}\left(\bar{\rho}_{g} \nu_{g} \widehat{D}_{g, i m}+\tau_{g, i j}\right)(46) \\
\frac{\partial}{\partial t} \bar{\rho}_{g} \widehat{E}_{g}+\frac{\partial}{\partial x_{m}}\left(\bar{\rho}_{g} \widehat{E}_{g}+\widehat{P}\right) \widehat{u}_{g, m} & =\Phi_{\rho_{g} E_{g}}+\frac{\partial}{\partial x_{m}}\left(\bar{\rho}_{g} \nu_{g} \widehat{D}_{g, i m} \widehat{u}_{g, i}+Q_{g, i j}\right)
\end{aligned}
$$

where $\rho_{g}, u_{g, i}, E_{g}, P$ and $\nu_{g}$ are the density, velocity, total energy, pressure and kinetic viscosity of the gas phase, $\widehat{D}_{g, i j}=\frac{\partial \widehat{u}_{l, i}}{\partial x_{j}}+\frac{\partial \widehat{u}_{l, i}}{\partial x_{j}}-\frac{2}{3} \frac{\partial \widehat{u}_{l, k}}{\partial x_{k}} \delta_{i j}$ is the strain tensor, $\tau_{g, i j}$ is the subgrid scale stress tensor modeled with the WALE approach [43], and $Q_{g, i j}$ is the filtered heat flux. $\Phi_{\rho_{g}}, \Phi_{\rho_{g} u_{g, i}}$ and $\Phi_{\rho_{g} E_{g}}$ are the coupling terms between liquid and gas phases. In the MEF, the coupling terms are:

$$
\begin{aligned}
\bar{\Phi}_{\rho_{g}} & =\overline{\dot{m}}_{l} \\
\bar{\Phi}_{\rho_{g} u_{g, i}} & =\overline{\dot{m}}_{l} \widehat{u}_{l, i}-\bar{F}_{d} \\
\bar{\Phi}_{\rho_{g} E_{g}} & =-\bar{F}_{d, i} u_{l, i}+\overline{\dot{m}}_{l}\left(\widehat{H}_{l}+\frac{1}{2} \widehat{u}_{l, i}^{2}\right)-\bar{\phi}_{l}
\end{aligned}
$$


whereas in the MMEF, the coupling terms are the sum of the contributions of each section:

$$
\begin{aligned}
\bar{\Phi}_{\rho_{g}} & =\sum_{k=1}^{N} E_{2}^{(k)} \bar{m}_{l}^{(k)} \\
\bar{\Phi}_{\rho_{g} u_{g, i}} & =\sum_{k=1}^{N}\left[-\bar{F}_{d, i}^{(k)}+E_{2}^{(k)} \bar{m}_{l}^{(k)} \widehat{u}_{l, i}^{(k)}\right] \\
\bar{\Phi}_{\rho_{g} E_{g}} & =\sum_{k=1}^{N}\left[-\bar{F}_{d, i}^{(k)} \widehat{u}_{l, i}+E_{2}^{(k)} \bar{m}_{l}^{(k)}\left(\widehat{H}_{l}^{(k)}+\frac{1}{2} \widehat{u}_{l, i}^{(k) 2}\right)-\bar{\phi}_{l}^{(k)}\right]
\end{aligned}
$$

In the Lagrangian approach, the coupling terms are obtained by simple projection on the gas flow grid [56]:

$$
\begin{aligned}
\bar{\Phi}_{\rho_{g}} & =-\sum_{p=1}^{N_{p}} \dot{m}_{p} \delta\left(\mathbf{x}-\mathbf{x}_{p}\right) \\
\bar{\Phi}_{\rho_{g} u_{g, i}} & =-\sum_{p=1}^{N_{p}}\left[\dot{m}_{p} u_{p, i}+F_{p, i}\right] \delta\left(\mathbf{x}-\mathbf{x}_{p}\right) \\
\bar{\Phi}_{\rho_{g} E_{g}} & =-\sum_{p=1}^{N_{p}}\left[F_{p, i} u_{p, i}+\frac{1}{2} \dot{m}_{p} u_{p, i}^{2}-\phi_{p}^{c}\right] \delta\left(\mathbf{x}-\mathbf{x}_{p}\right)
\end{aligned}
$$

where $N_{p}$ is the number of Lagrangian particles.

\section{The aeronautical-type configuration and numerical methods}

\subsection{The configuration}

The MERCATO test rig is an experimental swirl combustor (Fig. 1) operated at ONERA (Fauga), for which measurements are available [18]. Air is injected inside the plenum and enters the combustion chamber through the swirler. The liquid injection is located at the end of the swirler stage, and at the center of the swirled gaseous jet. The studied case is a two-phase evaporating flow without combustion.

The injection system is a pressure-swirl atomizer. It generates a hollow cone spray with a half angle of $40^{\circ}$ and orthoradial motion, which produces a polydisperse cloud of droplets. The droplet size distribution at $13 \mathrm{~mm}$ downstream the injector is shown in Fig. 3. The injected fuel is kerosene, modeled by a a surrogate [54] in the simulations.

The liquid flow rate is $1 \mathrm{~g} / \mathrm{s}$ at a temperature of $300 \mathrm{~K}$, and the gas flow rate is $15 \mathrm{~g} / \mathrm{s}$ at a temperature of $463 \mathrm{~K}$.

\subsection{Numerical methods}

\subsubsection{Gas phase}

The AVBP code solves compressible Navier-Stokes equation for reactive flows on unstructured grids in a cell-vertex formulation. To perform Large Eddy Simula- 


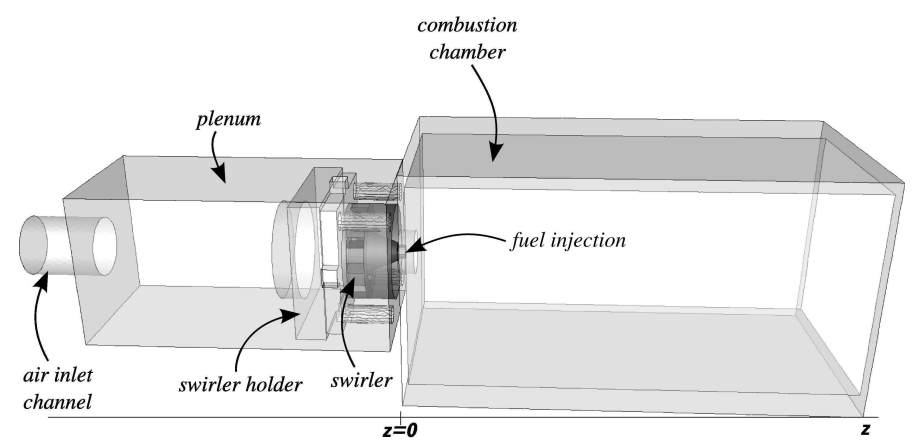

Fig. 1: Scheme of the MERCATO test rig.

tions, a $3^{\text {rd }}$ order in time and space finite element scheme is used [5]. To handle the oscillatory behavior of such a scheme, artificial viscosity [63] is added where the solution exhibits strong gradients. Non-reflecting NSCBC boundary conditions [45] and wall law [43] associated to the LES Smagorinsky model are used.

\subsubsection{Lagrangian disperse phase}

The Lagrangian equations are solved in AVBP using a first order Euler explicit time integration. Considering that the computation is driven by the acoustic time step, a first order in time scheme is sufficient. The interpolation of gas properties at particle positions is made by a linear least square approximation.

\subsubsection{Eulerian disperse phase}

The Eulerian liquid phase solver of AVBP is similar to the gas phase solver in terms of equations, but it behaves like a highly compressible flow, so that vacuum and strong gradients occur, difficult to handle numerically. A classical way to handle such numerical problems is to use artificial viscosity, associated to sensors that detect the difficulties. This methodology has been applied to the simulation of a decaying Homogeneous Isotropic Turbulence [59], and has proven to be very accurate. However more adapted schemes are needed in order to improve the robustness of the solver, like kinetic schemes [8], but their derivation in unstructured cell vertex 3-dimensional formulation is not straightforward and requires further developments.

\subsection{Boundary conditions for the disperse phase}

Injection is a keypoint in the simulation of two-phase flow burners. Here the injection system is modeled by the FIMUR model [54], which using autosimilarity assumptions. Fig. 2 shows a sketch of this model [54]. The model gives the following 


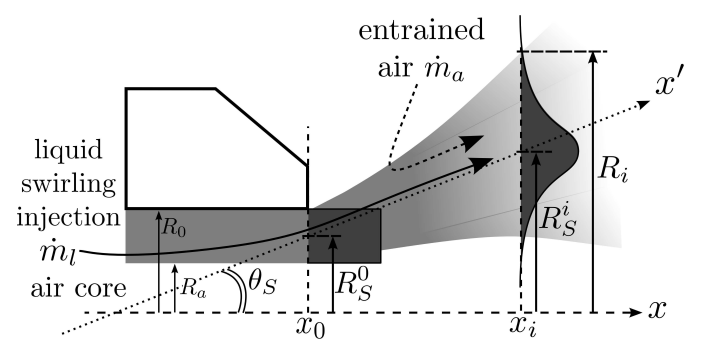

simplex

atomizer

model

EL simulation

analytical

model

Fig. 2: Definition of parameters and sketch of the FIMUR methodology [54].

injection condition in radial coordinates at fixed $x$ position:

$$
\begin{aligned}
u_{l, r}^{i}(\theta, r) & =\frac{\dot{m}_{l}}{\rho_{l} A_{p}} \sqrt{1-\left(\frac{R_{S}^{0}}{R_{S}^{i}}\right)^{2} \frac{r}{R_{S}^{i}}} \\
u_{l, \theta}^{i}(\theta, r) & =\frac{\dot{m}_{l}}{\rho_{l} A_{p}} \frac{R_{S}^{0}}{R_{S}^{i}} \frac{r}{R_{S}^{i}} \\
u_{l, x}^{i}(\theta, r) & =\frac{\dot{m}_{l}}{\pi R_{0}^{2}} A_{u} \\
m_{l}^{i}(\theta, r) & =\frac{\pi R_{0}^{2}}{I_{\alpha} A_{u}} \exp \left(\frac{-\left(r-R_{S}^{i}\right)^{2}}{\sigma^{2}}\right)
\end{aligned}
$$

where $R_{S}^{0}, R_{S}^{i}$ are the middle position of the liquid sheet at the injector exit and the liquid spray at the numerical injection location, $R_{0}$ the radius of the injection hole, $A_{p}$ the injection surface, $A_{u}$ a parameter which accounts for contraction effects on the discharge orifice as well as air entrainement effects along the axial distance and $\sigma$ the variance of the Gaussian profile of liquid mass. This model was initially designed for monodisperse injection, and its extension to multifluid injection is made by distributing the liquid volume fraction and droplet number density over all diameters, following the size distribution of Fig. 3. First the number density $f_{\text {num }}^{(p)}$ of each section is calculated by integrating the experimental size distribution $f_{\exp }$ over each interval:

$$
f_{n u m}^{(k)}=\int_{S^{(k)}}^{S^{(k+1)}} f_{e x p}\left(S_{p}\right) d S_{p}
$$

Then the mass density of each section is obtained as:

$$
f_{\text {mass }}^{(k)}=f_{\text {num }}^{(p)} \frac{\rho_{l}}{24 \sqrt{\pi}} \frac{\left(S^{(k+1) 2}-S^{(k) 2}\right)}{\left(\sqrt{S^{(k+1)}}-\sqrt{S^{(k)}}\right)}
$$

The total mass predicted by the monodisperse FIMUR model is then distributed using $f_{\text {mass }}^{(k)}$ :

$$
m_{l}^{(k), i}=f_{m a s s}^{(k)} m_{l}^{i}
$$


The velocity of each section is equal to the monodisperse velocity, in order to keep the same momentum for multifluid injection and monodisperse injection:

$$
\begin{aligned}
u_{l, r}^{(k), i}=u_{l, r}^{i}, \quad u_{l, \theta}^{(k), i} & =u_{l, \theta}^{i}, \quad u_{l, x}^{(k), i}=u_{l, x}^{i} \\
\sum u_{l, x}^{(k), i} m_{l}^{(p)} & =u_{l, x}^{i} \sum m_{l}^{(p)}=u_{l, x} m_{l}
\end{aligned}
$$

This assumption allows to compare monodisperse and polydisperse results with an equivalent injection condition in terms of velocity. To obtain a more accurate injection condition, it would be desirable to adapt the FIMUR model to a polydisperse spray. This will be a future work on this model.

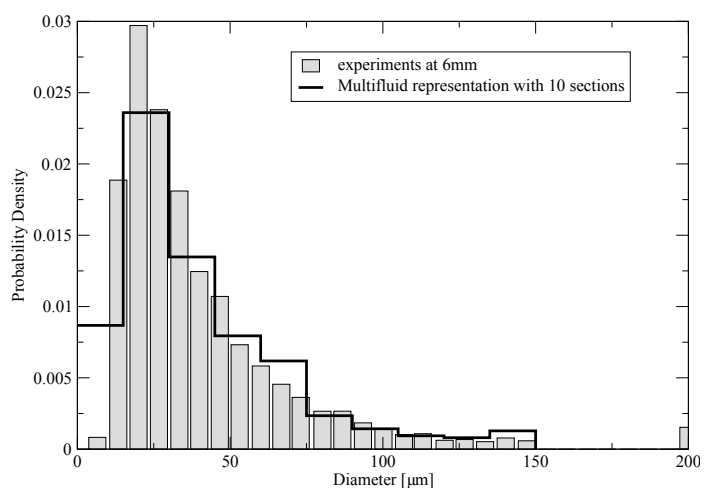

Fig. 3: MERCATO: Number Density Function at $13 \mathrm{~mm}$ downstream the injector location (histogram) and Multifluid representation with 10 sections (solid line).

\section{Importance of the polydispersion for the MERCATO configuration on reduced test configurations}

In this section, the importance of the polydispersion in the MERCATO conditions is investigated on two reduced configurations: a $0 \mathrm{D}$ evaporation case and a $2 \mathrm{D}$ frozen vortex. The former is expected to show the influence of the polydispersion on the evaporation time and the mean properties of the liquid phase, and the latter is expected to show its influence on the spatial dispersion induced by the drag-forced size-conditionned dynamics. The parameters of the two cases are set to represent the conditions in the MERCATO test rig.

\subsection{D Evaporation}

The initial temperature is $300 \mathrm{~K}$ for the droplets and $463 \mathrm{~K}$ for the gas phase. For the MEF simulations, the initial diameter is $55 \mu \mathrm{m}$. For the MMEF, the NDF is the one of Fig. 3. Because of transient heating, no analytical solution is available.

Figure 4 shows the time evolution of the total mass and mean temperature for MEF and MMEF. First, one can notice the important difference on the evaporation 
time for the two formalisms. Where the MEF exhibits an evaporation time of $50 \mathrm{~ms}$, the MMEF predicts an evaporation time of $400 \mathrm{~ms}$. This difference appears also on the heating time (time to reach the maximum temperature), for which the MEF predicts $25 \mathrm{~ms}$ whereas the MMEF predicts $150 \mathrm{~ms}$. Those two effects are directly related to the polydispersion, as the MEF cannot capture the large evaporation and heating times of big droplets.
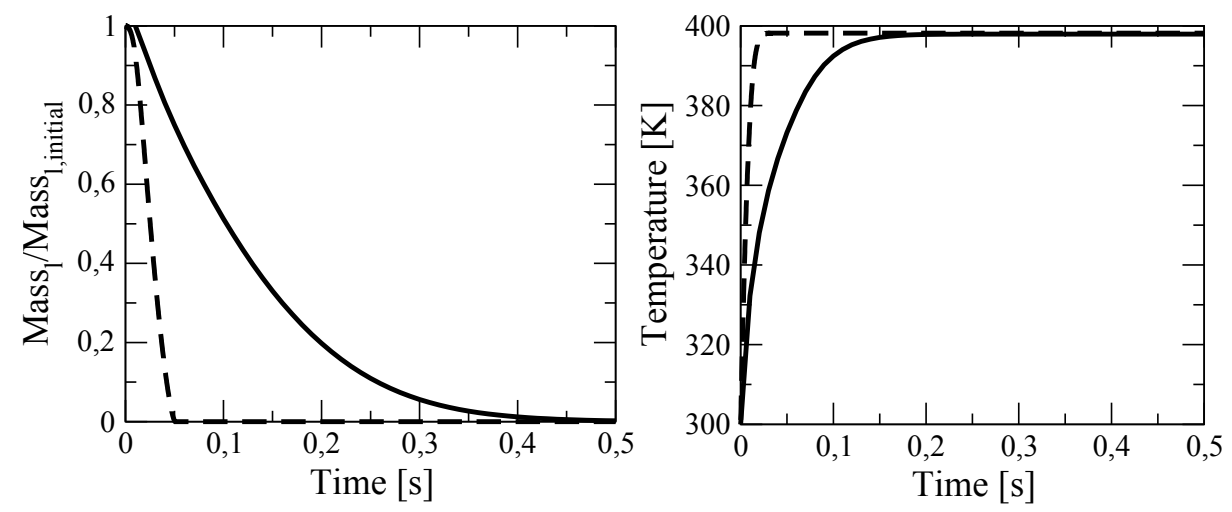

Fig. 4: Evaporation test case in MERCATO conditions: Evolution of total mass (upper left), droplet number density (upper right), liquid temperature (lower left) and mean diameter (lower right) for MMEF (full line) and MEF (dashed line).

\section{$4.22 \mathrm{D}$ Vortex}

It is proposed here to analyse the spatial dispersion effects of a $2 \mathrm{D}$ vortex having the same characteristics than the swirl motion in MERCATO at $13 \mathrm{~mm}$. The test case is a two-dimensional frozen vortex, with a spatially homogeneous initial droplet distribution having the same velocity as the gas phase. The radial velocity is zero and the tangential velocity of the gas phase corresponds to an isotropic vortex:

$$
U_{g, \theta}(r)=\frac{\Gamma r}{r_{v}^{2}} \exp \left(-\frac{r^{2}}{2 r_{v}^{2}}\right)
$$

where $\Gamma=0.6638$ is the vortex strength, and $r_{v}=0.012$ its radius. Figure 5 shows that Eq. 66 reproduces well the velocity profile of MERCATO. As the edge of the vortex has a non-zero radial velocity, Eq. 66 is used only for $r<0.02$. For $r \geq 0.02$, a linear interpolation of the true MERCATO profile is used.

The domain of $[-0.12,0.12]^{2} \mathrm{~m}$ is discretized with $800^{2}$ quad cells. Ten sections are used for the MMEF, with the same droplet number in each section. The computation is run for $5 \mathrm{~ms}$, which is the characteristic time needed by the injected spray to impact the wall in the MERCATO configuration.

On Fig. 6, the total droplet number density obtained at $5 \mathrm{~ms}$ is shown for MEF and MMEF. The high vorticity of the central zone ejects the droplets with a force depending on their sizes. With the MEF approach, an accumulation zone is 


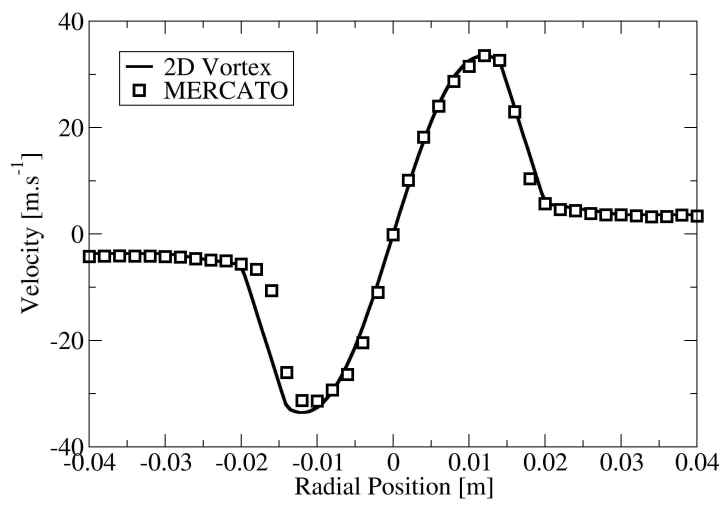

Fig. 5: Gas velocity profile for the 2D vortex (solid line) and in MERCATO test case (13mm downstream the injection location)(squares).

generated, which corresponds to the accumulation of droplets in the low vorticity zone, latter convected due to the non-zero velocity at the edge of the vortex. With the MMEF approach, one can see several accumulation zones, each corresponding to one section, with its proper Stokes number and so its proper dynamics.

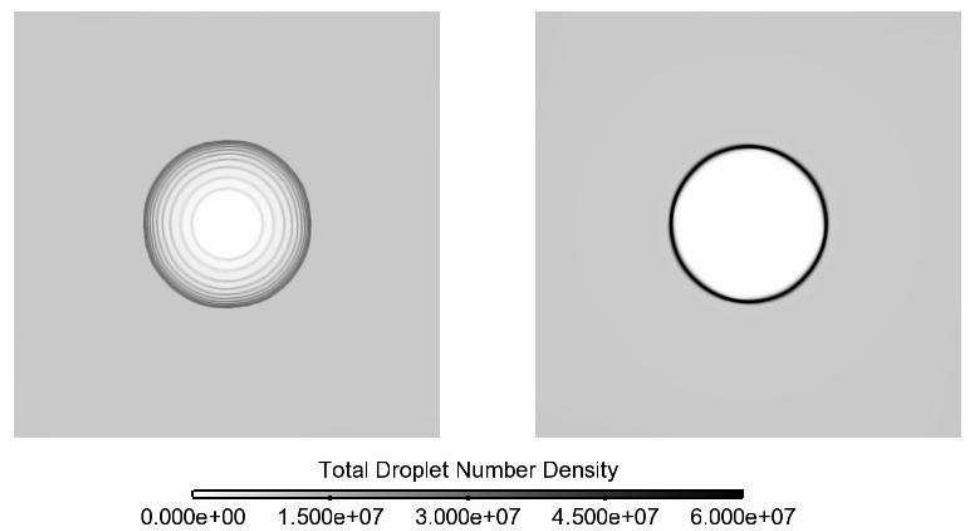

Fig. 6: Droplet Number Density at $t=5 \mathrm{~ms}$ for MMEF (left) and MEF(right).

Figure 7 shows the number density and mean diameter radial profiles for both MEF and MMEF at $5 \mathrm{~ms}$. While the droplet number density is widely distributed spatially in MMEF, it is concentrated around $0.038 \mathrm{~m}$ in MEF. Due do the weaker influence of the gas phase, bigger droplets keep their initial velocity out of the vortex, and so their initial radial deviation. On the contrary, small droplets are in equilibrium with the gas phase, and reach a small velocity out of the vortex. This impacts the mean diameter profile, with small diameters near the vortex 
center and bigger diameters far from it. Two difficulties are shown in Fig.7. The first one is linked to the mean diameter at the center of the vortex, where the absence of droplets should lead to a zero mean diameter. However the numerical methods impose to keep a non-zero droplet number density everywhere, even in empty zones where it is set to a value several orders of magnitude lower than the mean value, leading to a non-zero mean diameter in these empty zones. The second difficulty concerns the discretization in sections, resulting in discrete accumulations corresponding to each section trajectories. However in practical cases, it is expected that turbulent mixing and species diffusion will considerably reduce this effect.

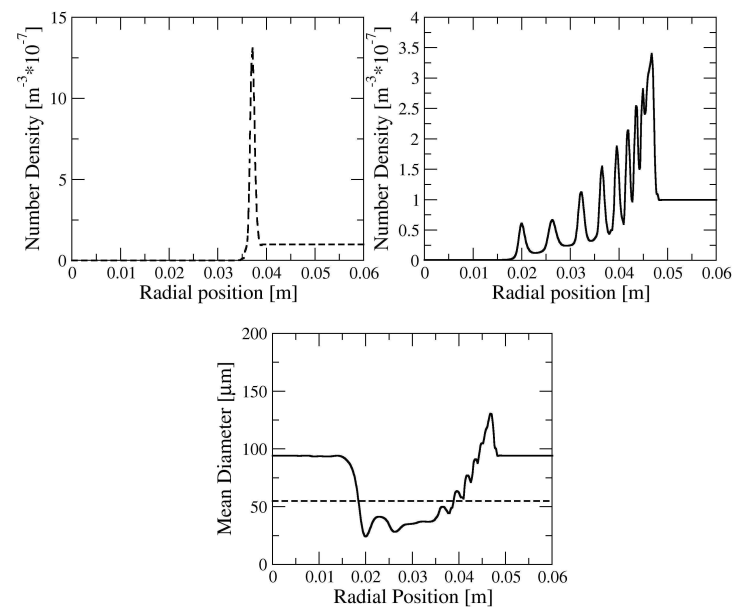

Fig. 7: Radial distribution of droplet number density for MEF (top left) and MMEF (top right), and arithmetic mean diameter (bottom) for MEF (dashed line) and MMEF (solid line).

\section{Results on the MERCATO configuration}

Here are compared the Lagrangian results of [56], the MEF results of [54] and the present MMEF results. For the MMEF approach, the mesh is composed of 1,351,767 tetrahedrons (Fig. 8), while 3,527,637 tetrahedrons were used for the MEF approach and 3,934,364 tetrahedrons were used for the Lagrangian. The main difference between these meshes concerns the region far from the injection (after $56 \mathrm{~mm}$ downstream the injection), all meshes having the same level of refinement in the region close to the injection location.

First the gas phase is validated on a pure gaseous computation (no disperse phase), verifying that the conditioning flow field for the dispersed phase is well resolved. Then liquid phase results are analyzed, qualitatively in terms of liquid volume fraction and gas mass fraction, and quantitatively in terms of mean and RMS velocity at 3 positions in the chamber. Size-conditioned statistics are all also investigated at 8 locations in the spray. 

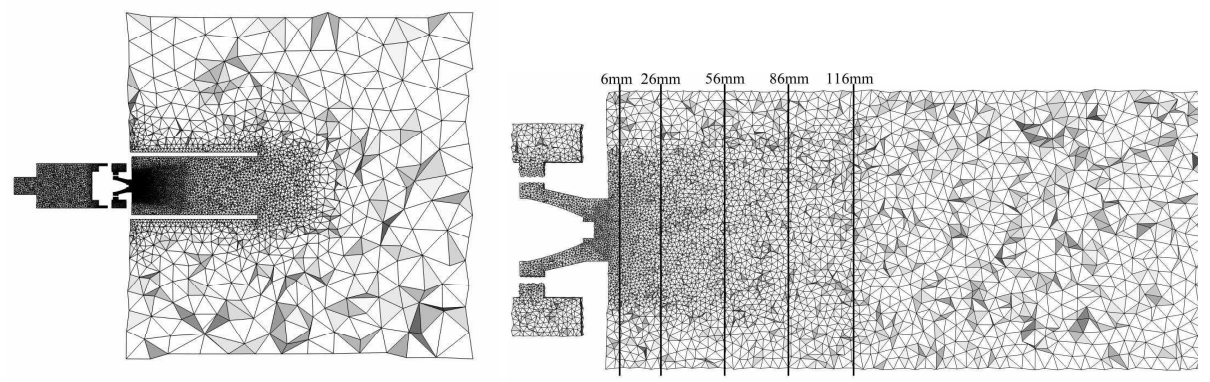

Fig. 8: Cut plane of the mesh of the combustion chamber

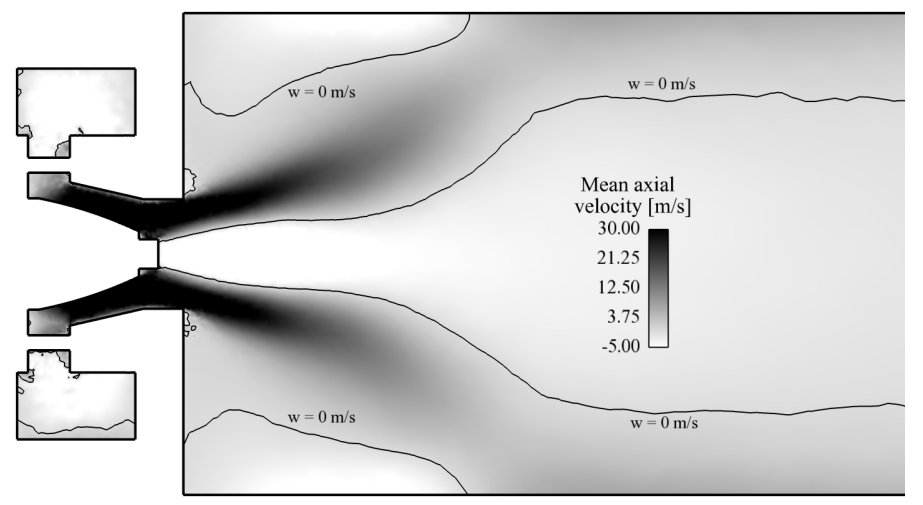

Fig. 9: Cut plane of the mean velocity field for the gas phase with isolines of zero axial velocity.

\subsection{Gas phase}

Mean quantities for the gas phase are obtained after averaging over $800 \mathrm{~ms}$. Figure 9 shows a cut plane of the mean axial velocity, with isolines of zero axial velocity. The flow exhibits two recirculation zones : the Central Toroidal Recirculation Zone (CTRZ) and the Corner Recirculation Zone (CRZ). One can also notice the high shear stress zone induced by the gas injection in the combustion chamber, which impacts on the wall, and follows it until the exit of the combustion chamber.

Figures 10-15 show mean and RMS velocites for axial, radial and orthoradial velocity components. Globally, numerical results are in good agreement with experiment for the first three measurement positions $(6,26$ and $56 \mathrm{~mm})$, where liquid phae measurements were made, whereas the RMS and velocities are underestimated far from the injection location $(86 \mathrm{~mm}$ and $116 \mathrm{~mm})$, due to the coarser mesh. The present results are considered sufficiently accurate to allow the spray modeling study. A more detailed characterization of the gas phase is available in [53] and [56]. 
Gaseous mean axial velocity $[\mathrm{m} / \mathrm{s}]$

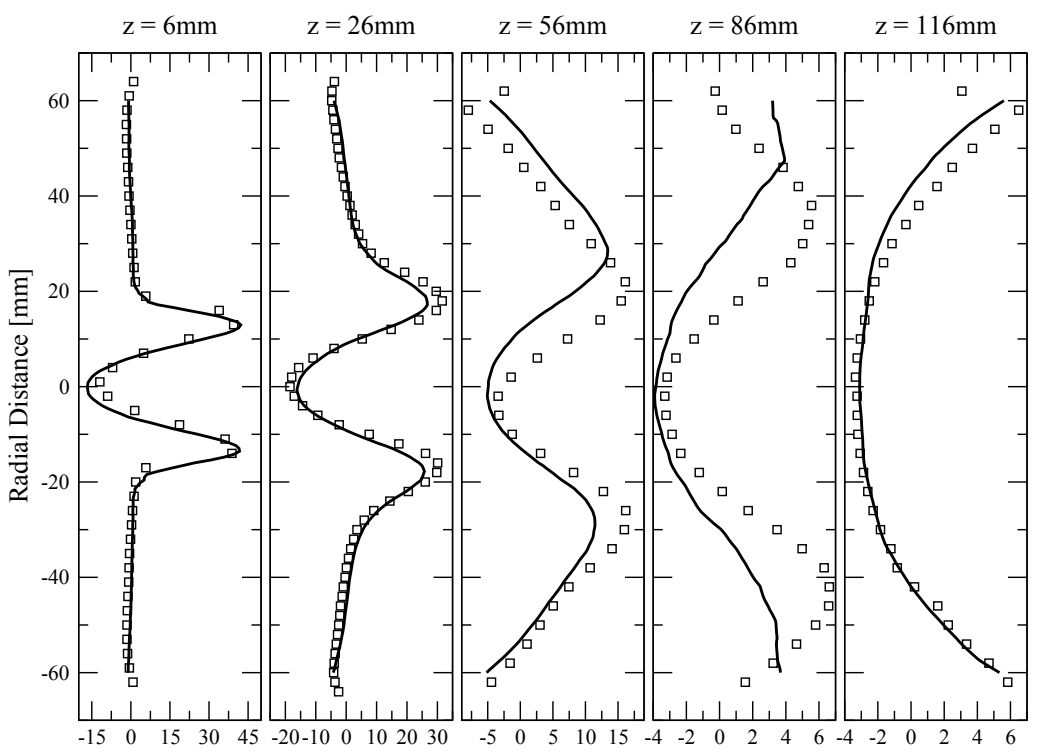

Fig. 10: Axial mean velocity profiles for the gas phase at $6,26,56,86$ and $116 \mathrm{~mm}$ locations (Fig. 8): experiment (square) and simulation (full line)

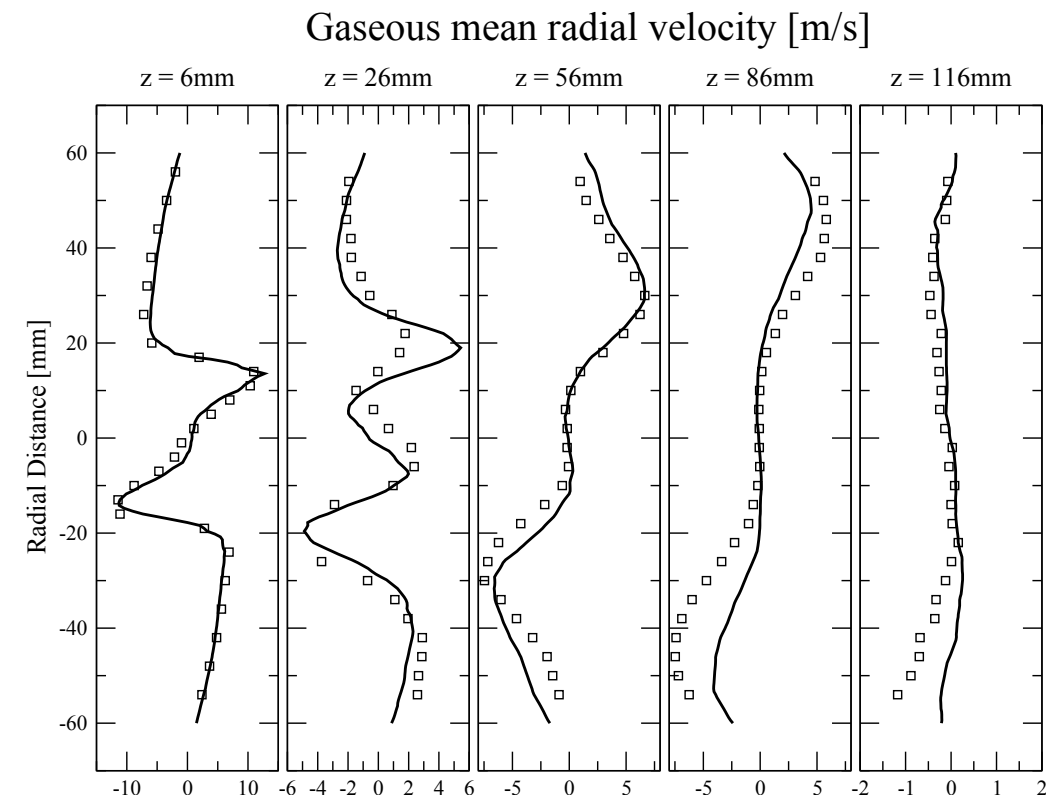

Fig. 11: Radial mean velocity profiles for the gas phase at $6,26,56,86$ and $116 \mathrm{~mm}$ locations (Fig. 8): experiment (square) and simulation (full line) 
Gaseous mean ortho-radial velocity $[\mathrm{m} / \mathrm{s}]$

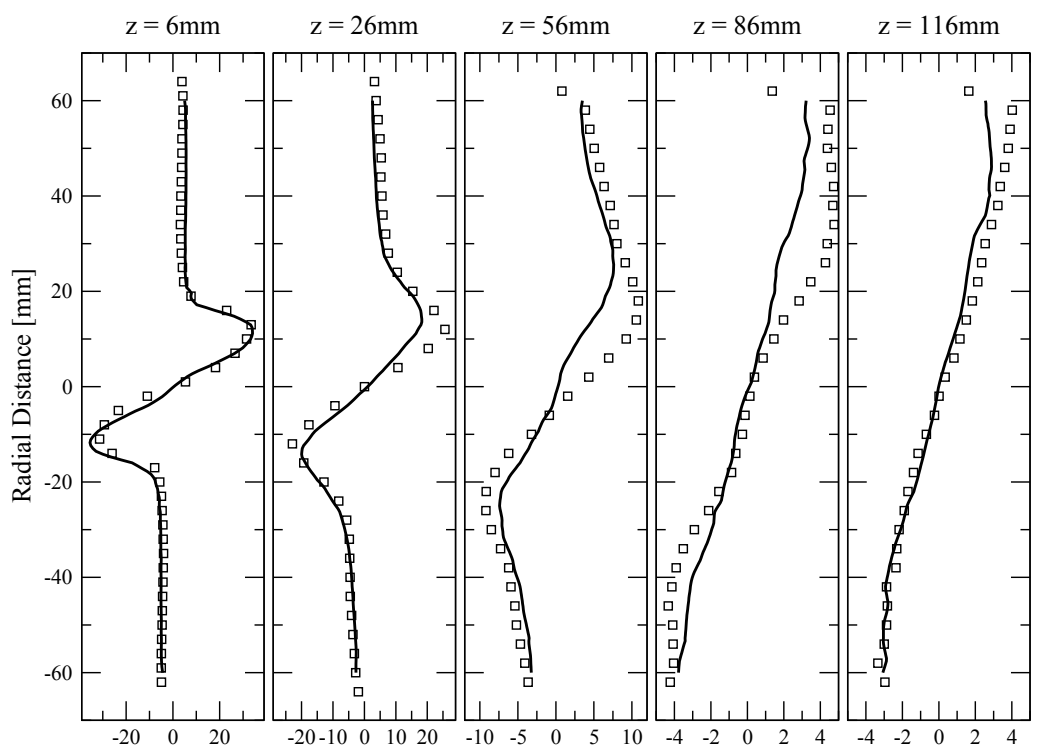

Fig. 12: Orthoradial mean velocity profiles for the gas phase at $6,26,56,86$ and $116 \mathrm{~mm}$ locations (Fig. 8): experiment (square) and simulation (full line)

\section{Gaseous RMS axial velocity [m/s]}

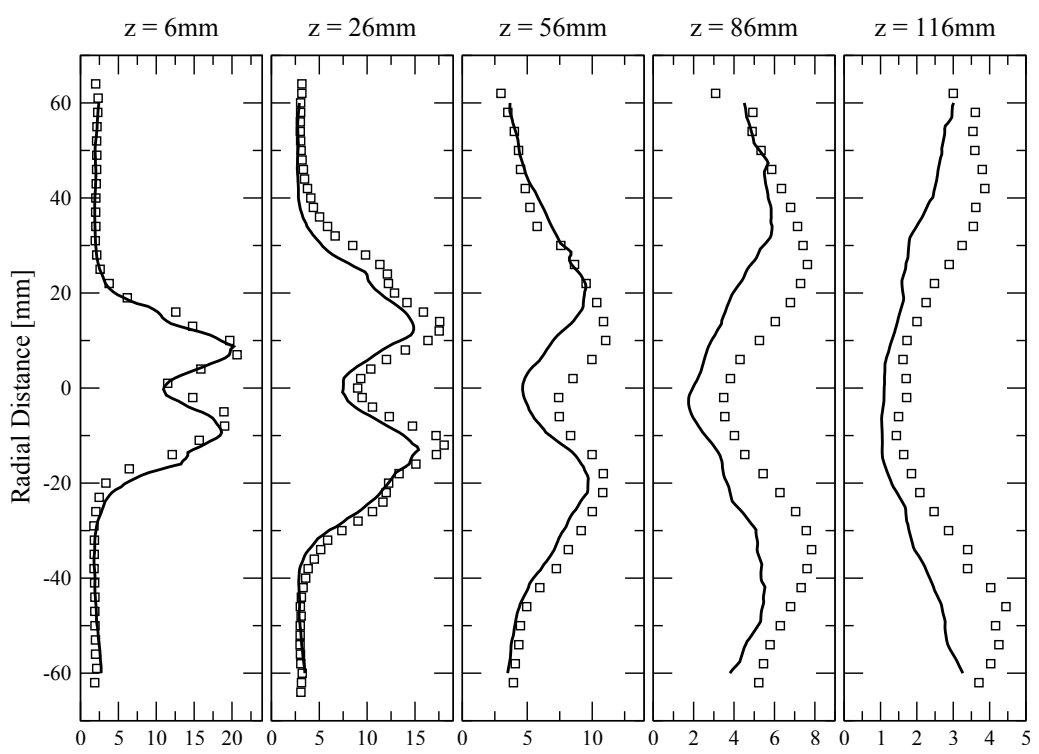

Fig. 13: Axial RMS velocity profiles for the gas phase at $6,26,56,86$ and $116 \mathrm{~mm}$ locations (Fig. 8): experiment (square) and simulation (full line) 


\section{Gaseous RMS radial velocity $[\mathrm{m} / \mathrm{s}]$}

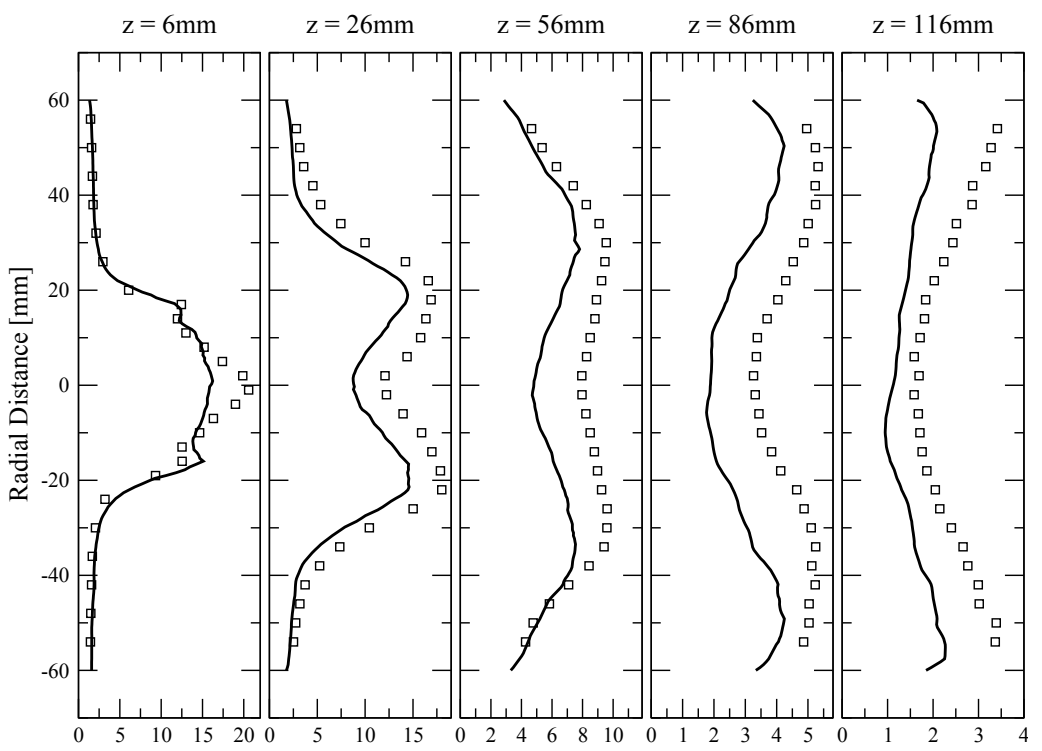

Fig. 14: Radial RMS velocity profiles for the gas phase at 6, 26, 56, 86 and $116 \mathrm{~mm}$ locations (Fig. 8): experiment (square) and simulation (full line)

Gaseous RMS ortho-radial velocity $[\mathrm{m} / \mathrm{s}]$

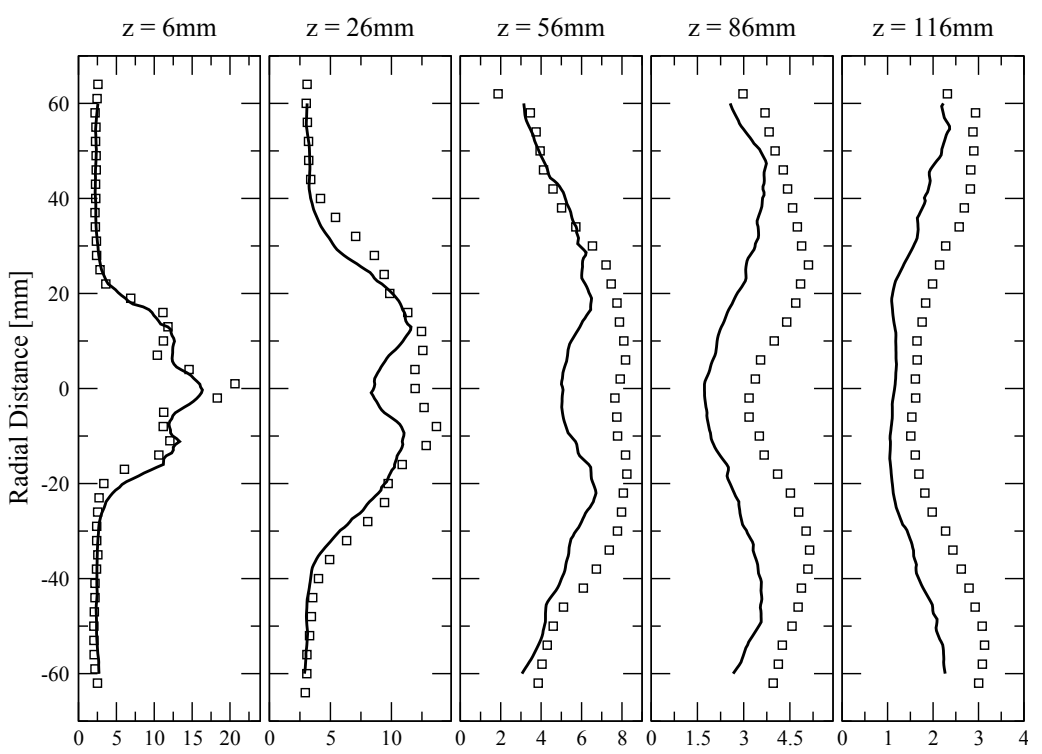

Fig. 15: Orthoradial RMS velocity profiles for the gas phase at 6, 26, 56, 86 and $116 \mathrm{~mm}$ locations (Fig. 8): experiment (square) and simulation (full line) 


\subsection{Liquid phase}

\subsubsection{Mean flow}

The results of EL [56], MEF [53, 54] and MMEF are confronted to experiment.

Figure 16 shows the mean liquid volume fraction for the three simulations. The three approaches exhibit similar distributions. The liquid spray impacts the wall at nearly the same position. Droplets are ejected by the CTRZ, leading to a zone empty of droplets in the center of the chamber. As expected, Eulerian cases appear more diffusive than the Lagrangian case. The major difference is linked to polydispersion: in EL and MMEF, large droplets persist in the whole chamber, while in MEF (monodisperse), the mean droplet lifetime is too short to allow them to reach the downstream zone.

Figures 17-19 show mean velocity profiles at three locations for the three simulations and the experiment. All results are globally in good agreement with experiment. Some discrepancies are observed in the central zone for the axial velocity and at the spray boundary for the radial velocity. The two polydisperse approaches (MMEF and EL) misestimate the axial velocity in the central zone, whereas the MEF reproduces it accurately. On the contrary, MEF predicts non-zero values near the spray boundary at $z=26 \mathrm{~mm}$, where MMEF and EL show values close to zero, in agreement with experiment. However discrepancies are small and all these results confirm that the size distribution has a weak effect on the mean velocity, as mentioned in [56].

Figures 20-22 shows RMS velocity profiles at the same locations. Here polydispersion has a more important effect. All models are similar and good close to the injection, but MEF underestimates the RMS velocities at further locations, while the two polydisperse approaches (MMEF and EL) give better predictions even downstream. Better results are obtained by the MMEF compared to EL, except for the orthoradial component in the last plane. This is possibly due to the way the RMS velocity is estimated. Indeed the RMS velocity is composed of the fluctuating part of the resolved field, the subgrid velocity and the random uncorrelated velocity. The latter is evaluated assuming isotropy, which is not true in shear flows, as shown in [38]. A better estimation of RMS velocities in MEF and MMEF would be achieved by defining the orientation of the stress tensor, but this is not investigated here. 


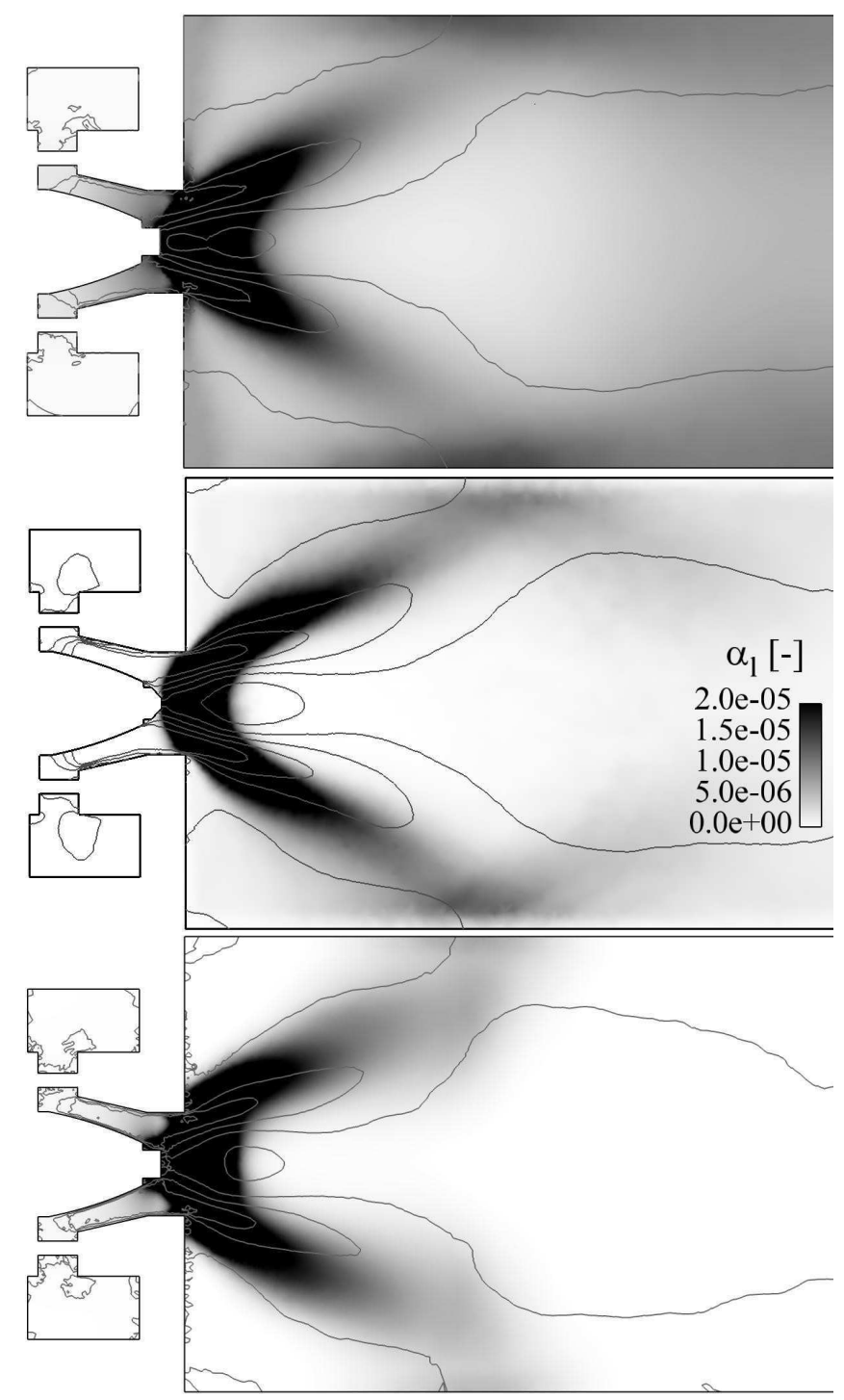

Fig. 16: Mean liquid volume fraction for MMEF (top), EL (middle) and MEF (bottom). 
Liquid mean axial velocity $[\mathrm{m} / \mathrm{s}]$

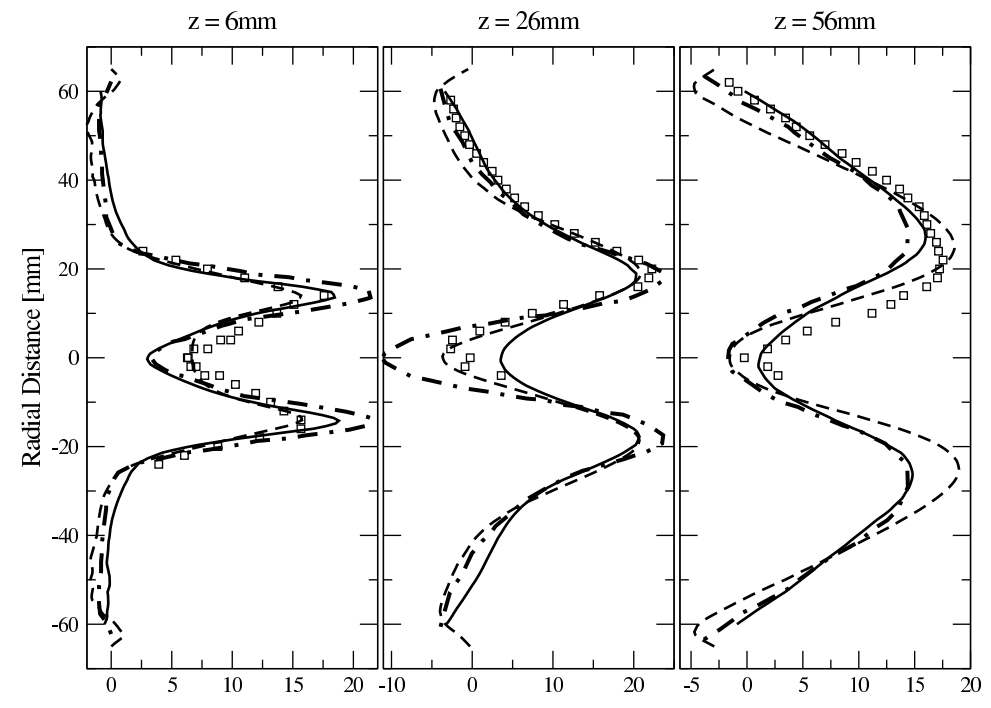

Fig. 17: Axial mean velocity profiles for the liquid phase at 6,26 and $56 \mathrm{~mm}$ locations (Fig. 8): experiment (square), MMEF (full line), MEF (dashed line) and EL (dot-dashed line).

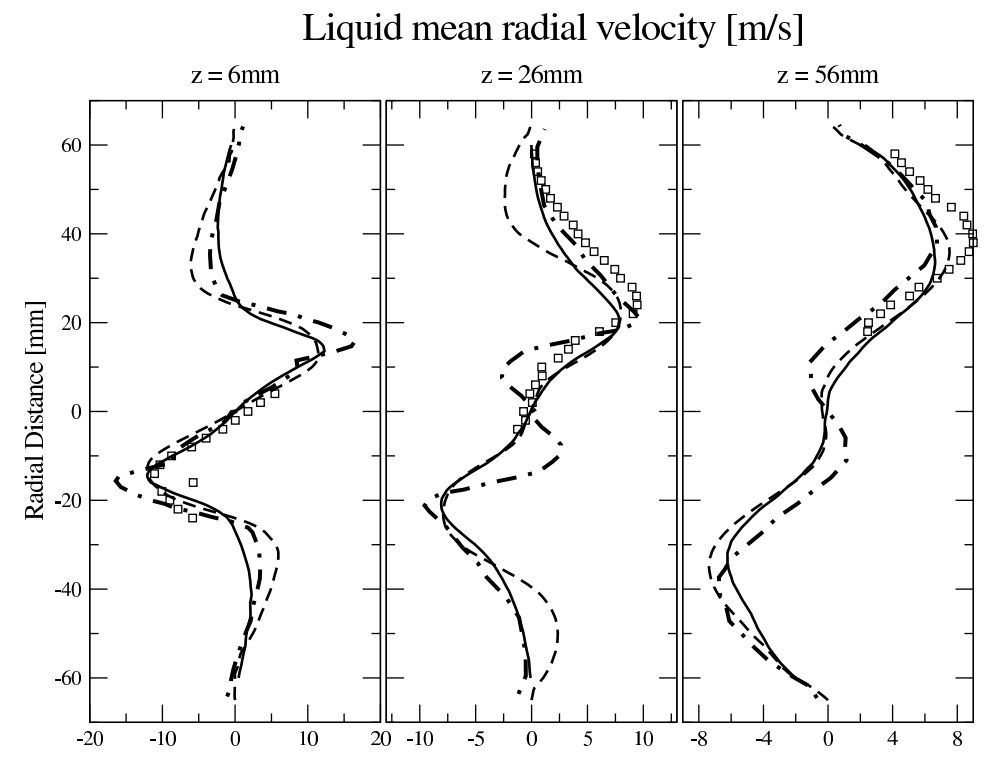

Fig. 18: Radial mean velocity profiles for the liquid phase at 6,26 and $56 \mathrm{~mm}$ locations (Fig. 8): experiment (square), MMEF (full line), MEF (dashed line) and EL (dot-dashed line). 
Liquid mean orthoradial velocity $[\mathrm{m} / \mathrm{s}]$

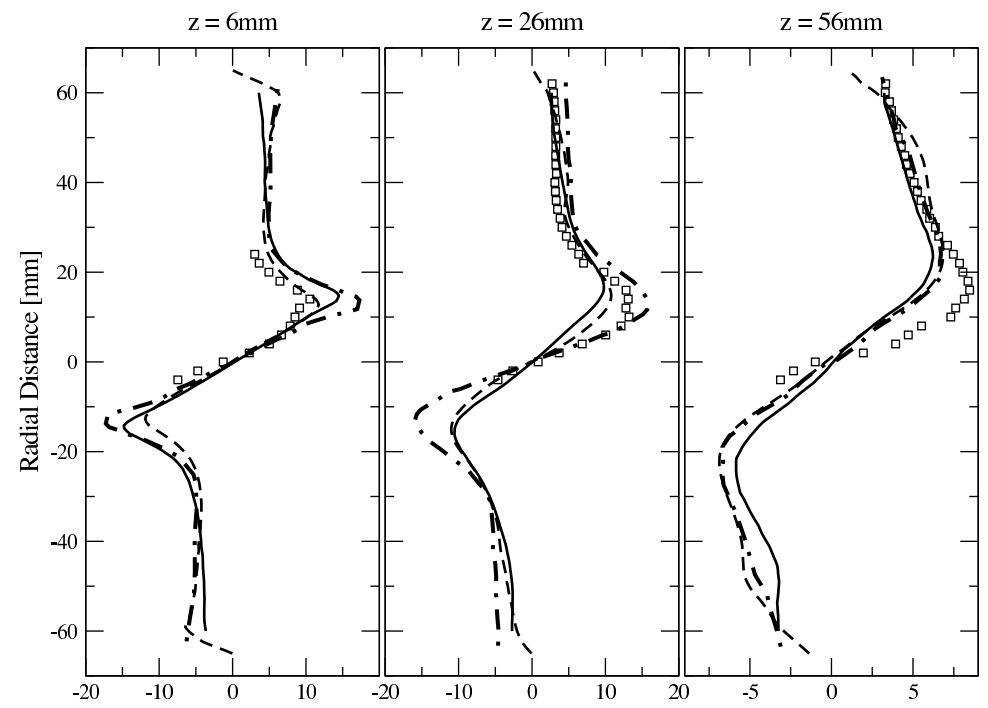

Fig. 19: Orthoradial mean velocity profiles for the liquid phase at 6,26 and $56 \mathrm{~mm}$ locations (Fig. 8): experiment (square), MMEF (full line), MEF (dashed line) and EL (dot-dashed line).

\section{Liquid RMS axial velocity $[\mathrm{m} / \mathrm{s}]$}

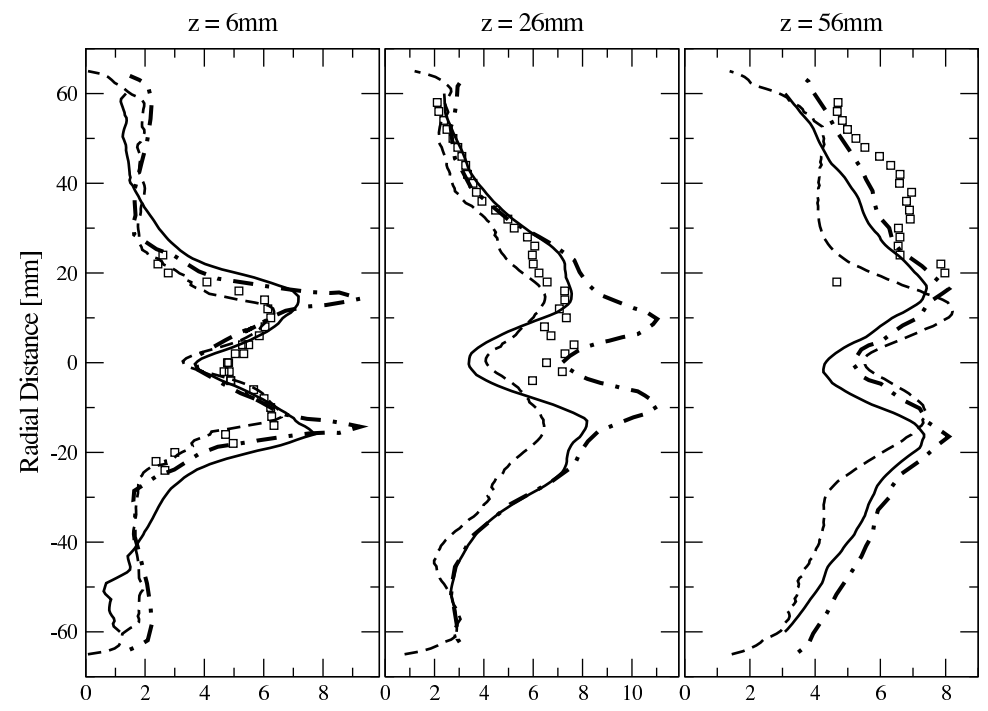

Fig. 20: Axial RMS velocity profiles for the liquid phase at 6, 26 and $56 \mathrm{~mm}$ locations (Fig. 8): experiment (square), MMEF (full line), MEF (dashed line) and EL (dot-dashed line). 
Liquid RMS radial velocity $[\mathrm{m} / \mathrm{s}]$

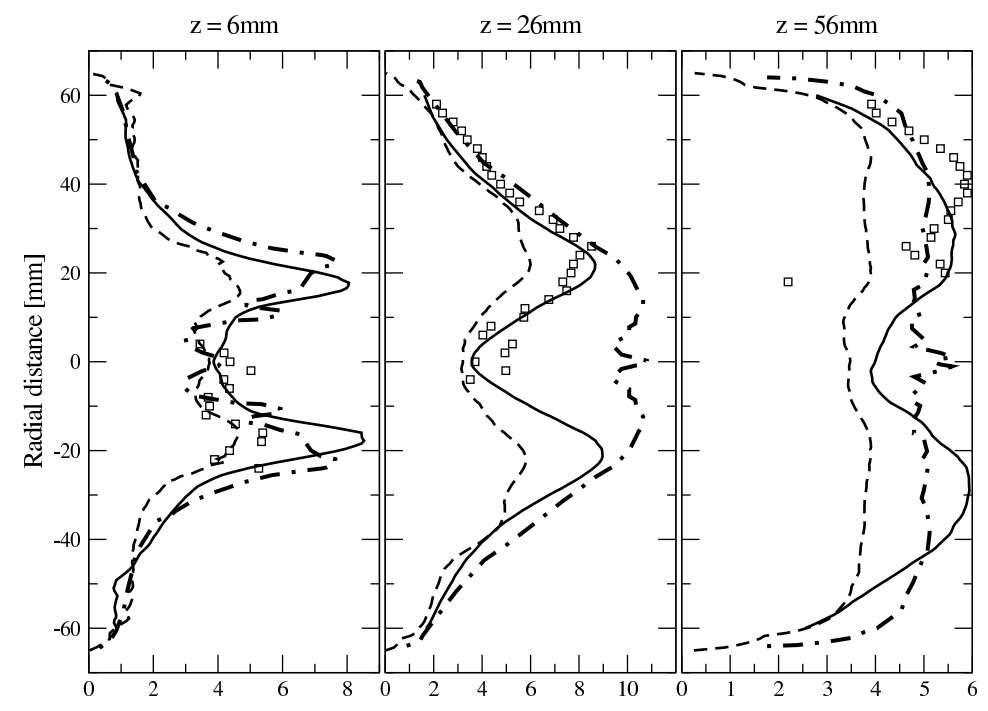

Fig. 21: Radial RMS velocity profiles for the liquid phase at 6,26 and $56 \mathrm{~mm}$ locations (Fig. 8): experiment (square), MMEF (full line), MEF (dashed line) and EL (dot-dashed line).

\section{Liquid RMS orthoradial velocity $[\mathrm{m} / \mathrm{s}]$}

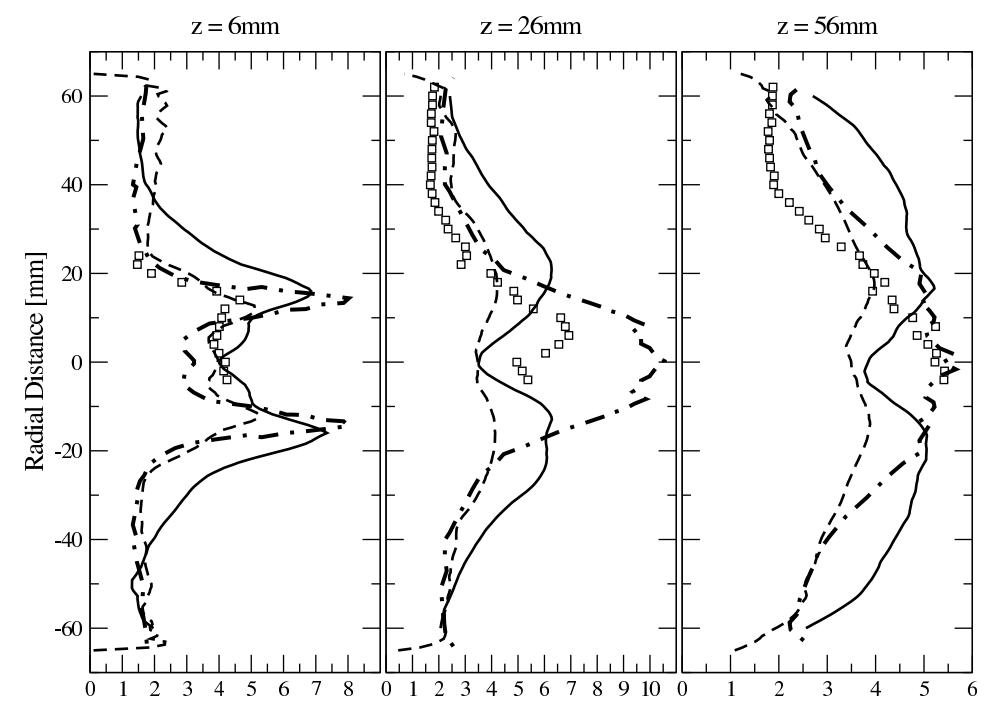

Fig. 22: Orthoradial RMS velocity profiles for the liquid phase at 6, 26 and $56 \mathrm{~mm}$ locations (Fig. 8): experiment (square), MMEF (full line), MEF (dashed line) and EL (dot-dashed line). 


\subsubsection{Size-conditioned statistics}

Size-conditioned statistics are investigated here for MMEF and EL at select locations, and compared to experiment. Table. 1 and Fig. 23 show the probe positions: three are on the axis (A1-A3), three in the spray (S1-S3), one at the spray boundary (B1) and one close to the wall (W3).

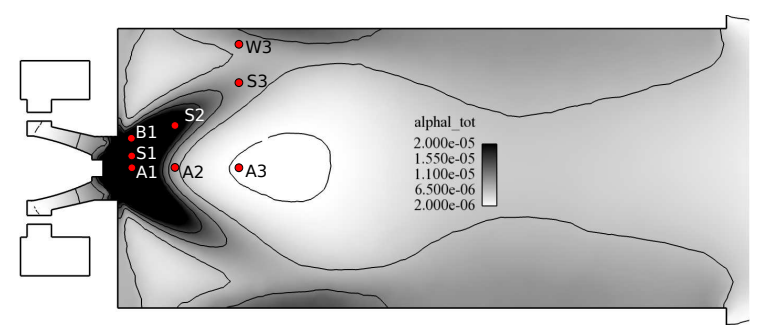

Fig. 23: Measurement position for size-conditioned statistics.

\begin{tabular}{ccccccccc} 
coordinate & A1 & S1 & B1 & A2 & S2 & A3 & S3 & W3 \\
\hline axial $(\mathrm{mm})$ & 6 & 6 & 6 & 26 & 26 & 56 & 56 & 56 \\
\hline radial $(\mathrm{mm})$ & 0 & 8 & 14 & 0 & 20 & 0 & 40 & 58 \\
\hline
\end{tabular}

Table 1: Probe location for size-conditioned statistics.

In Fig. 24, MMEF and EL size distributions are compared to experimental results. At $26 \mathrm{~mm}$ and $56 \mathrm{~mm}$, EL and MMEF are in good agreement with experiment. At $6 \mathrm{~mm}$, both approaches reproduce the size distribution at the boundary of the spray, but not on the axis and in the spray, where the distribution is shifted towards big sizes, with worse results for the MMEF. This may be due to the absence of secondary break-up model, as shown by Senoner et al. [56]. One can also notice that MMEF overestimates the probability of big droplets, which may be due to the absence of secondary break-up, which is suppose to break these big droplets, but can also be related to the phase space diffusion occuring in such a first order Multifluid approach, and resulting in overestimated mean sizes [32].

Figures 25-27 show size-conditioned velocities. The axial component is relatively well captured by both approaches. Some discrepancy is observed for the velocities of small droplets at $6 \mathrm{~mm}$, on the axis, where both simulations predict a high negative velocity, whereas the experiment show a positive velocity. Considering that small droplets have a strong tendency to follow the gas phase, and that the gas phase velocity is about $-15 \mathrm{~m} / \mathrm{s}$ at this location, the experimental results are surprising. A possible explanation of such a behavior is that the liquid injection strongly modifies the gas flow close to the injector, due to a dense and coherent liquid core, shifting the CTRZ downstream and thus leading to positive velocities at the first measurement location. As we inject a dispersed phase directly, the impact will be limited compared to a dense structure, and a modification of the FIMUR 
model may be needed in this perspective. For radial and orthoradial components, EL and MMEF are in good agreement with experiment. Largest differences are found at $6 \mathrm{~mm}$ on the axis, where MMEF predicts a quasi-zero velocity for those two components, while EL shows high velocities of small droplets. These two results differ from the experimental results, and may also be explained by a lack of the injection strategy. It is interesting to notice that experiment and EL computations suffer from a poor statistical convergence on the axis where the droplet flux is low, whereas the statistical description of both eulerian formalisms (MEF and MMEF) achieves converged statistics.

The axial velocity conditioned by size (Fig. 25) also gives informations about the spatial dispersion of droplets. Basically, velocities on the axis (A1-A3) shows that droplets smaller than $30 \mu \mathrm{m}$ are sensitive to the CTRZ, as they always have a negative axial velocity, contrary to droplets bigger than $30 \mu \mathrm{m}$, which have a positive axial velocity. Furthermore, the axial velocities at the point W3 show that droplets smaller than $30 \mu m$ are also captured by the CRZ. 

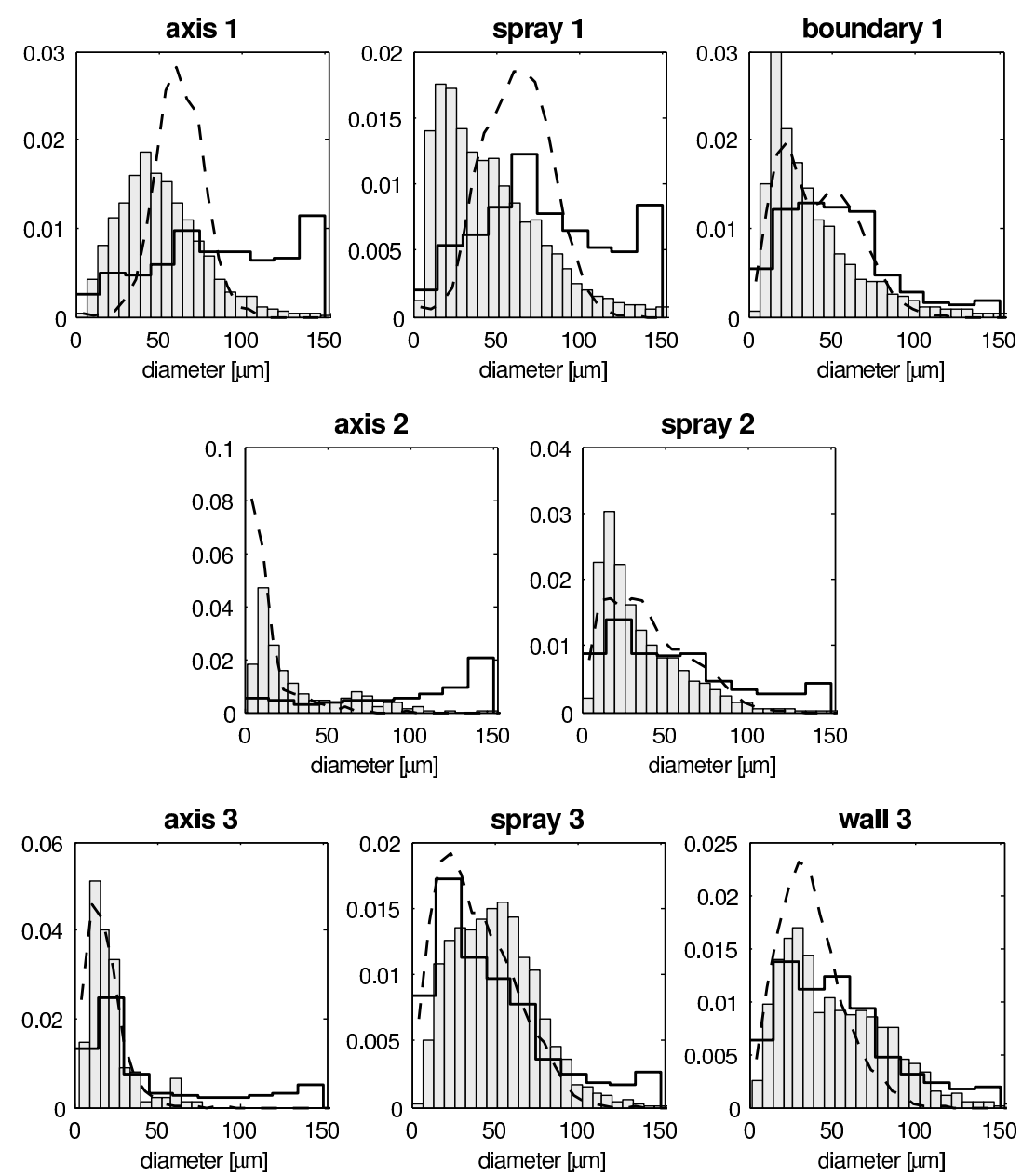

Fig. 24: Size distributions: experiment (histograms), MMEF (full line) and EL (dashed line). 

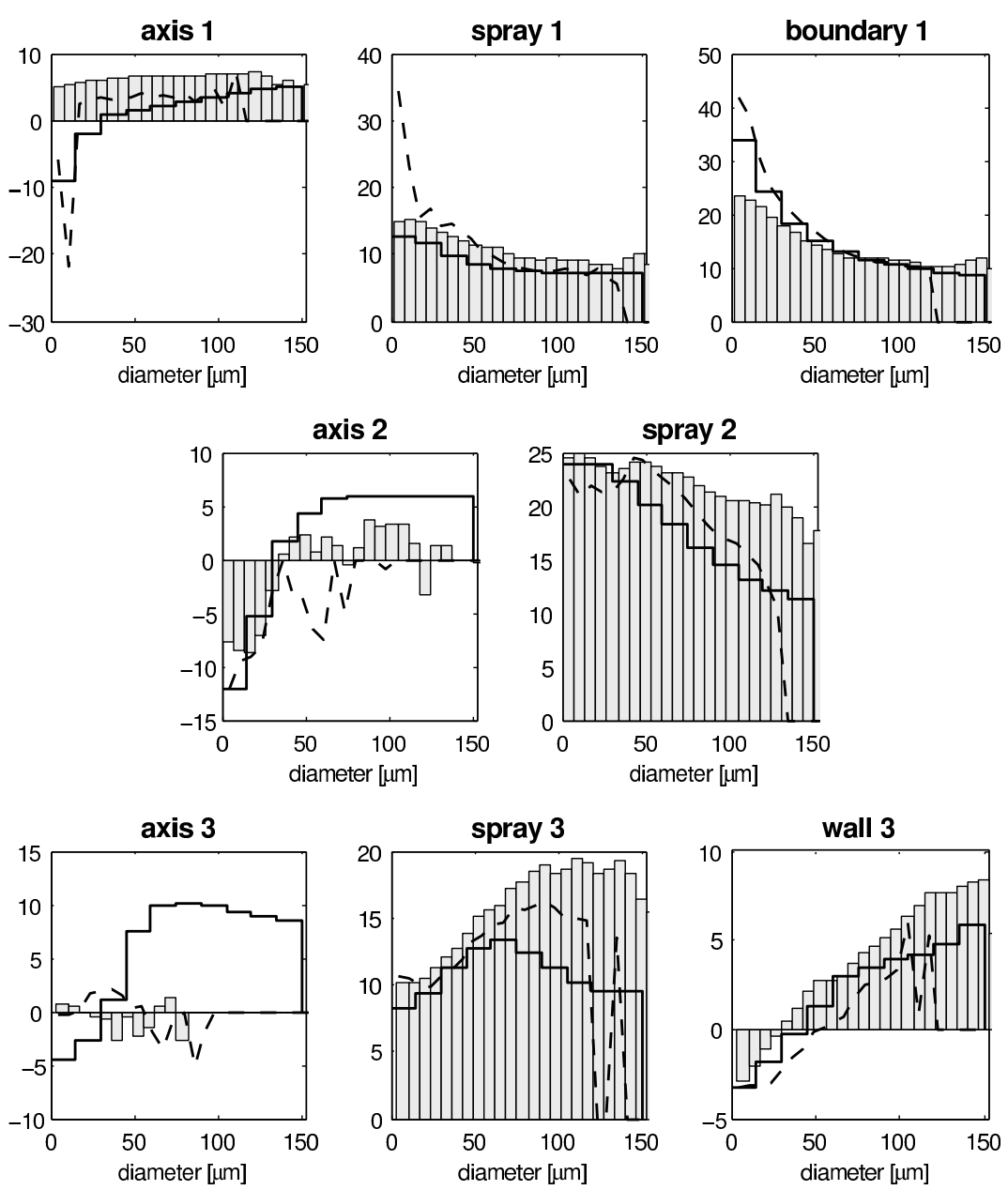

Fig. 25: Size-conditioned axial velocity: experiments (histograms), MMEF (full line) and EL (dashed line). 

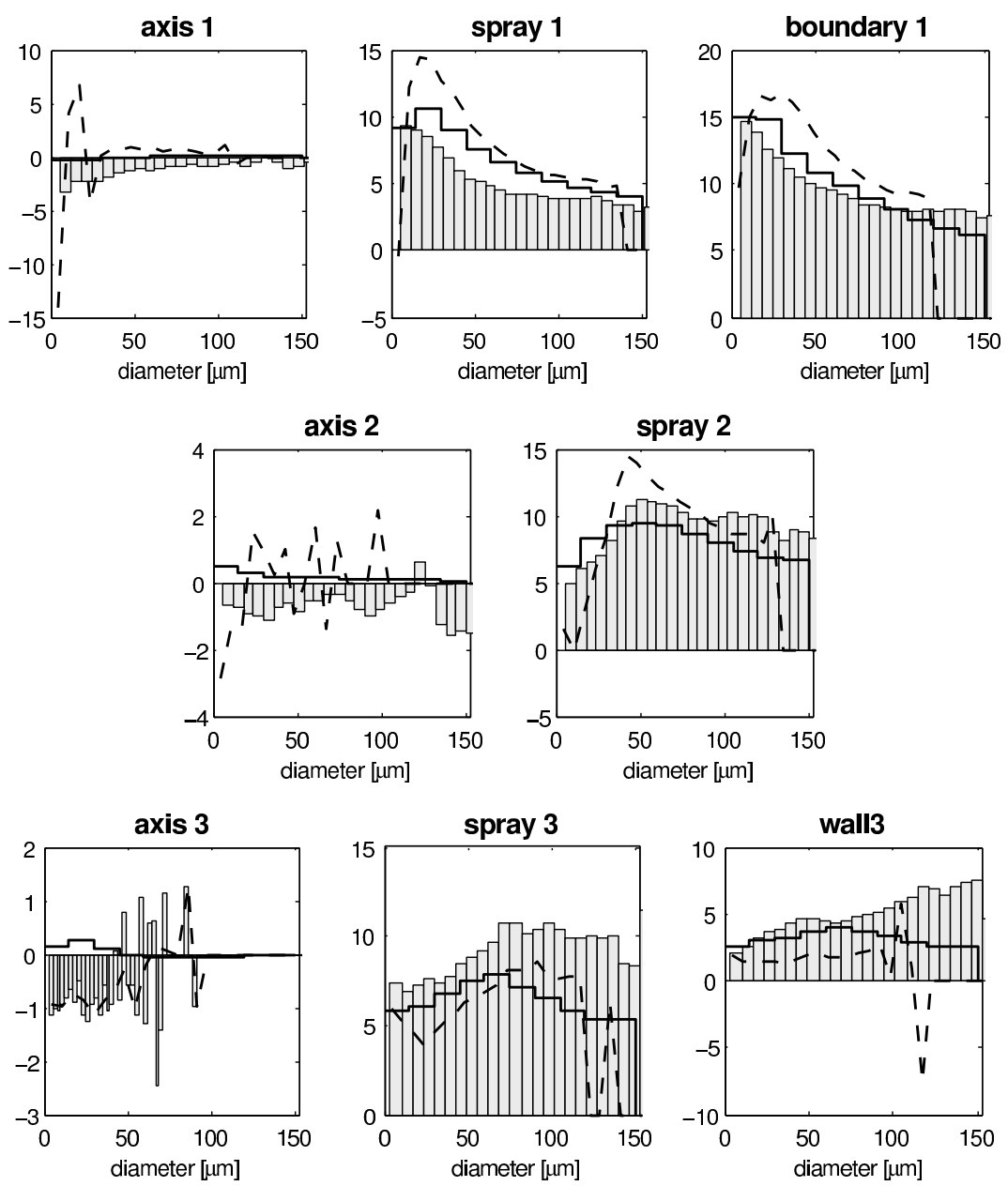

Fig. 26: Size-conditioned radial velocity: experiments (histograms), MMEF (full line) and EL (dashed line). 

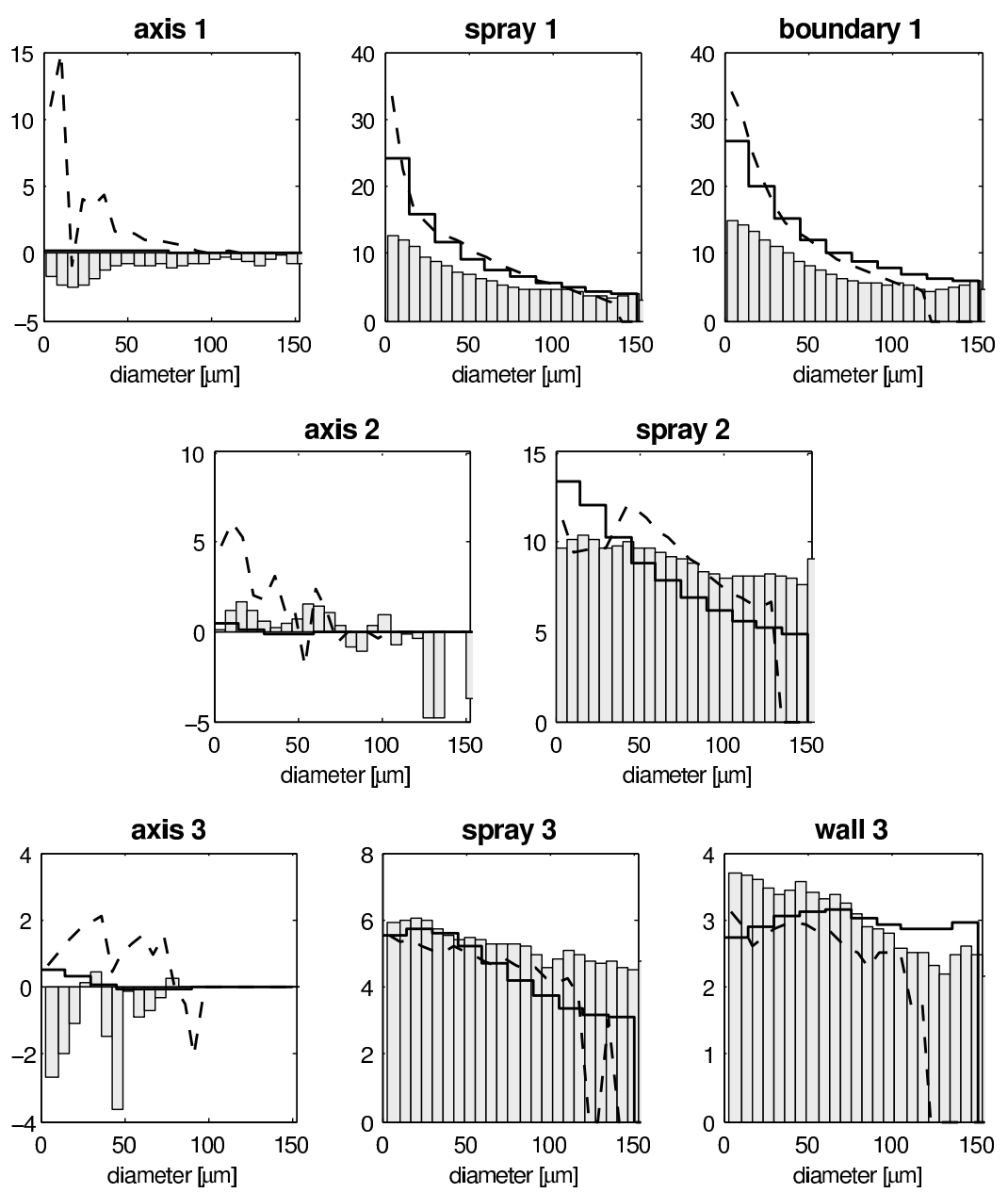

Fig. 27: Size-conditioned orthoradial velocity: experiments (histograms), MMEF (full line) and EL (dashed line). 


\subsubsection{Gaseous kerosene mass fraction}

On Fig. 28, instantaneous gaseous kerosene mass fraction for MEF, MMEF and EL are compared. No experimental results are available but the three simulations show a great influence of the polydispersity on the kerosene distribution. In particular, MEF predicts a high mass fraction close to the injection, whereas the two polydisperse approaches lead to a higher mass fraction downstream the combustion chamber. This is again due to the shorter life time of the mean size droplets in the monodisperse approach, leading to the complete disappearance of the liquid phase at $60 \mathrm{~mm}$ downstream the injection location. These results in conjunction with the liquid phase distribution will lead to a different behavior in terms of ignition and combustion $[60,4]$.

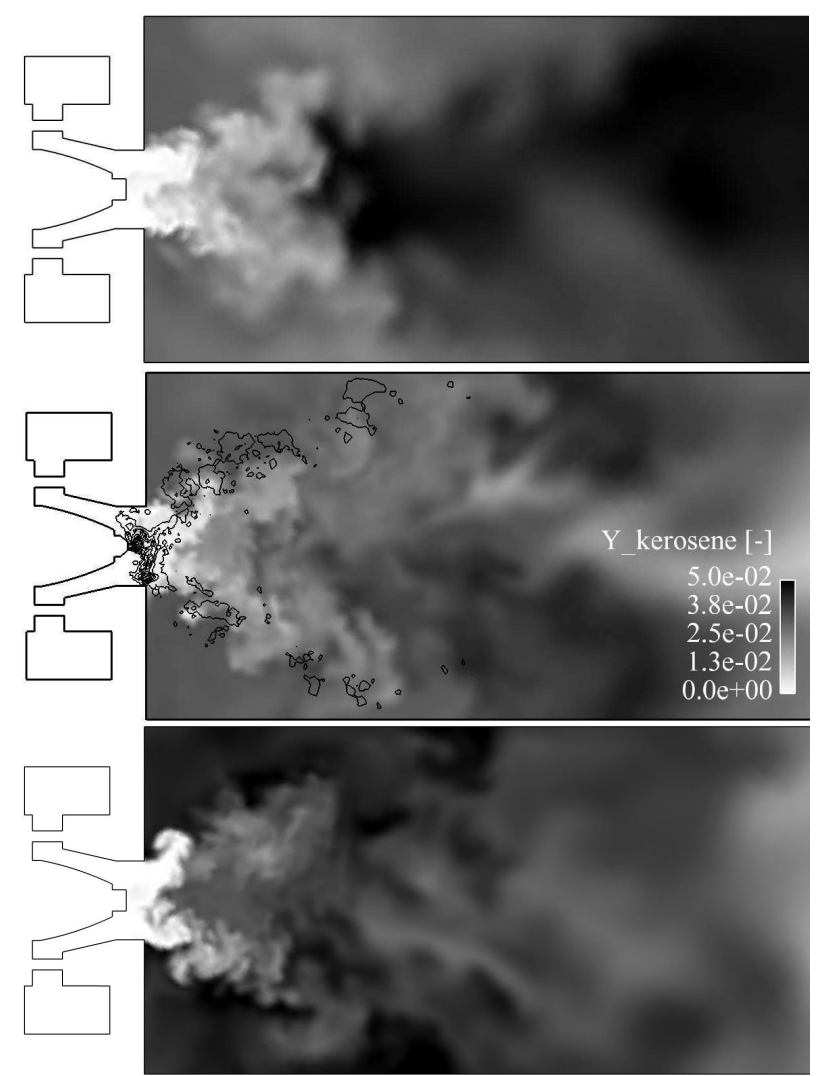

Fig. 28: Instantaneous kerosene mass fraction for MMEF (top), EL (middle, image from [56], black lines represent iso-lines of mass transfer) and MEF

(bottom). 


\section{Conclusions}

Accounting for polydispersion in Eulerian-Eulerian simulation of turbulent twophase flows has been proposed by combining the mesoscopic Eulerian formalism [13] and the Multifluid Approach [31]. The resulting Multifluid Mesoscopic Eulerian Formalism is able to capture size-conditioned statistics on drag, turbulence and evaporation. This formalism has been integrated in the AVBP solver and applied to the simulation of an aeronautical test rig operated by ONERA and called MERCATO [18].

The gaseous flow is first validated and results are in good agreement with the experiment for the three first measurement planes.

Results on the two-phase flow are compared to experiment and to two other modeling approaches: the monodisperse mesoscopic Eulerian formalism and a polydisperse Eulerian-Lagrangian approach.

Mean liquid velocities are well captured by all approaches, confirming the weak influence of polydispersity on these quantities, as stated in [56]. RMS velocities are however significantly impacted by polydispersity, as shown by the good agreement obtained with the two polydisperse approaches (EL and MMEF), where the MEF tends to underestimate RMS velocities.

Results in terms of size-conditioned statistics show a good agreement of both approaches with experiment, even if some discrepancies appear close to the injection. The resulting field of gaseous kerosene mass fraction confirms the great influence of polydispersity, showing a totally different repartition between polydisperse and monodisperse approaches. This is critical for the prediction of ignition and combustion, and comparison with experiment is crucially needed here.

Finally, even if results obtained by EL and MMEF are similar, it is important to note that the computational time required by the 10 sections MMEF simulation is about six times higher than the EL simulation with 285000 particles (MEF and EL simulations require approximatively the same resources for this configuration [53]). This overcost could be reduced by using moment methods such as high order multifluid approaches [32, 10]. An interesting alternative is to use moments methods like the Coupled Size-Velocity Moments method (CSVM) of Vié et al. [62]. This model is an extension of the Eulerian Multi-Size Moment method (EMSM) of Kah et al. [25, 40], which captures the evaporation process more accurately than the 10 sections multifluid approach with only 4 size moments. It enables to capture size-velocity correlations, which permits to reconstruct the velocity for each droplet size, and thus is of primary importance in real flows like the MERCATO configuration. Its adaptation to turbulent regimes and implementation in AVBP will be interesting for complex industrial simulations.

\section{Acknowledgments}

We would like to thanks Marlène Sanjosé and J.M. Senoner for providing their numerical set-up and results for the MERCATO configuration, and C. Angelberger and S. De Chaisemartin for several fruitful discussions. We also acknowledge the computational resources of IFP énergies nouvelles and CERFACS where simulations were performed. 


\section{References}

1. Apte S.V., Mahesh K., Moin P. and Oefelein J.C., Large-eddy simulation of evaporating spray in a coaxial combustor, Proc. Combust. Inst. 32, 2247-2256, 2009

2. Belt R.J. and Simonin O., Quadrature method of moments for the pdf modeling of droplet coalescence in turbulent two-phase flow, Proceedings of the ASME FEDSM 2009, FEDSM2009-78095, 2009

3. Bird G.A., Molecular gas dynamics and the direct simulation of gas flows, Oxford Science Publications, 42, 1994

4. Boileau M., Simulations aux grandes échelles de l'allumage diphasique des foyers aéronautiques, Ph.D. Thesis, Institut National Polytechnique de Toulouse, 2007, available on http://ethesis.inp-toulouse.fr/archive/00000558/

5. Colin 0. and Rudgyard M., Development of high-order Taylor-Galerkin schemes for unsteady calculations, Journal of Computational Physics, Vol. 162(2), pp. $338-371,2000$

6. Cossali G.E., An integral model for gas entrainment into full cone sprays, Journal of Fluid Mechanics, Vol. 439(2), pp. 353-366, 2001

7. Crowe, C.T., Sommerfeld, M. and Tsuji, Y., Multiphase Flows with Droplets and Particles, CRC Press LLC, Boca Raton, FL, 1998

8. De Chaisemartin S., Modèles eulériens et simulation de la dispersion turbulente de brouillards qui s'évaporent, Ph.D. Thesis, Ecole Centrale Paris, 2009, available on TEL http://tel.archives-ouvertes.fr/tel-00443982/en

9. Desjardins O., Moureau V. and Pitsch H., An accurate conservative level set /ghost fluid method for simulating turbulent atomization, Journal of Computational Physics, 227(18): 8395-8416, 2008

10. Dufour G. and Villedieu P., A second-order multi-fluid model for evaporationg sprays, Mathematical Modelling and Numerical Analysis, Vol. 39(5), pp. 931-963, 2005

11. Drew, D. and Passman, S., Theory of multicomponent fluids, Applied Mathematical Sciences, Vol.135, 1999

12. Ferry, J. and Balachandar, S., Equilibrium Eulerian approach for predicting the thermal field of a dispersion of small particles, International Journal of Heat and Mass Transfer, Vol. 48, pp. 681-689, 2005

13. Février P., Simonin O. and Squires K.D., Partitioning of particle velocities in gas-solid turbulent flow into a continuous field and a spatially uncorrelated random distribution: theoretical formalism and numerical study, Journal of Fluid Mechanics, Vol. 533, pp. 1-46, 2005

14. Frössling, Über die Verdunstung fallender Tropfen (On the evaporation of falling drops), Gerlands Beitr. Geophys., Vol. 52, pp. 170-216, 1938

15. Fox R.O., Laurent F. and Massot M., Numerical simulation of spray coalescence in an Eulerian framework: Direct quadrature method of moments and multi-fluid method, Journal of Computational Physics, Vol. 227, pp. 3058-3088, 2008

16. Fox R.O., A quadrature-based third-order moment method for dilute gasparticle flow, Journal of Computational Physics, Vol. 227, pp. 6313-6350, 2008

17. Garcia M., Development and validation of the Euler-Lagrange formulation on a parallel and unstructured solver for large-eddy simulation, Ph.D. Thesis, Université de Toulouse, Toulouse, 2009, available on TEL http://tel.archives- 
ouvertes.fr/tel-00414067/en/

18. Garcia-Rosa N., Phénomènes d'allumage d'un foyer de turbomachine en conditions de haute altitude, Ph.D. Thesis, Université de Toulouse, Toulouse, 2008 19. Primary Atomizing Modeling, Annual Review of Fluid Mechanics, Vol. 40, pp. 343-366, 2008

20. Greenberg J.B., Silverman I. and Tambour Y., On the origin of spray sectional conservation equations, Combustion and Flame, Vol. 93, pp. 90-96, 1993

21. Hubbard, G.L., Denny, V.E. and Mills, A.F., Droplet evaporation: effects of transient and variable properties, Int.J. Heat Mass Transf., Vol. 18, pp. 10031008,1975

22. Jaegle F., Large eddy simulation of evaporating sprays in complex geometries using Eulerian and Lagrangian methods, Ph.D. Thesis, Université de Toulouse, 2009, available on http://ethesis.inp-toulouse.fr/archive/00001024/

23. F. Jaegle, J.-M. Senoner, M. Garcia, F. Bismes, R. Lecourt, B. Cuenot and T. Poinsot, Eulerian and Lagrangian spray simulations of an aeronautical multipoint injector , Proceedings of the Combustion Institute, 33(2):2099 - 2107, 2011

24. Kah D., Laurent F., Massot M. and Jay S., Modeling of polydisperse sprays using a high order method for numerical advection of size moments, 11th International Conference of Liquid Atomization and Spray Systems, Vail, USA, July 2009

25. Kah, D., Taking into account polydispersity in the framework of a coupled Euler-Lagrange approach for the modeling of liquid fuel injection in internal combustion engines, Ph.D. Thesis, Ecole Centrale Paris, 2010, available online at http://tel.archives-ouvertes.fr/tel-00618786/en/

26. Kah D., Laurent F., Massot M. and Jay S., A high order moment method simulating evaporation and advection of a polydisperse spray, In Press, Journal of Computational Physics, 2011, doi:10.1016/j.jcp.2011.08.032

27. Kaufmann A., Moreau M., Simonin O. and Helie J., Comparison between Lagrangian and mesoscopic Eulerian modelling approaches for inertial particles suspended in decaying isotropic turbulence, Journal of Computational Physics, Vol. 227(13), pp. 6448-6472, 2008

28. Kuo K., Principles of Combustion, Wiley-Interscience, New York, 2nd edition, 2005

29. Lamarque, N., Schémas numériques et conditions limites pour la simulation aux grandes echelles de la combustion diphasique dans les foyers d'hélicoptre, Ph.D. Thesis, Institut National Polytechnique de Toulouse, 2007, available on http://ethesis.inp-toulouse.fr/archive/00000593/

30. Laget O., Reveille B., Habchi C., Martinez L., Truffin K., and Angelberger C., LES Calculations of a four cylinder engine, SAE Detroit, 2011

31. Laurent F. and Massot M., Multi-fluid modeling of laminar poly-dispersed spray flames: origin, assumptions and comparison of sectional and sampling methods, Combustion Theory and Modelling, Vol. 5, pp. 537-572, 2001

32. Laurent F., Numerical analysis of Eulerian multi-fluid models in the context of kinetic formulations for dilute evaporating sprays, Mathematical Modeling and Numerical Analysis, Vol. 3, pp. 431-468, 2006

33. McGraw R., Description of aerosol dynamics by the quadrature method of moments, Aerosol Science and Technology, Vol. 27, pp. 255-265, 1997 
34. Mahesh K., Constantinescu G. and Moin P., A numerical method for large eddy simulation in complex geometries, Journal of Computational Physics, 197 (1): 431-468, 2004

35. Martinez L., Vié A., Jay S., Benkenida, A. and Cuenot B., Large eddy simulation of fuel sprays using the Eulerian mesoscopic approach. validations in realistic engine conditions, 11th International Conference of Liquid Atomization and Spray Systems, Vail, USA, July 2009

36. Martinez L., Simulation aux grandes échelles de l'injection de carburant liquide dans les moteurs combustion interne , Ph.D. Thesis, Université de Toulouse, 2009, available on http://ethesis.inp-toulouse.fr/archive/00000934/

37. Martinez L., Benkenida, A. and Cuenot, B., A model for the injection boundary conditions in the context of 3D simulation of diesel spray : methodology and validation, Fuel, 89 (1): 219-228, 2010

38. Masi E., Simonin O. and Bédat, B., The mesoscopic Eulerian approach for evaporating droplets interacting with turbulent flows, Flow Turbulence and Combustion, 86, pp 563-583, 2011

39. Massot M., Knikker R., Péra C. and Réveillon J., Lagrangian/Eulerian analysis of the dispersion of evaporating droplets in a non-homogeneous turbulent flow, International Conference on Multiphase Flow, Yokohama, Japan, 2004

40. Massot M., Laurent F., Kah D. and de Chaisemartin S., A robust moment method for evaluation of the disappearance rate of evaporating sprays, SIAM Journal of Applied Mathematics, Vol. 70, pp. 3203-3234, 2010, available on HAL http://hal.archives-ouvertes.fr/hal-00332423/en/

41. Ménard T., Tanguy S. and Berlemont A., Coupling level set/VOF/ghost fluid methods: Validation and application to 3D simulation of the primary break-up of a liquid jet, International Journal of Multiphase Flow, 33(5): 510-524, 2007

42. Moreau M., Bédat B. and Simonin O., Development of gas-particle eulereuler LES approach: a priori analysis of particle sub-grid models in homogeneous isotropic turbulence, Flow Turbulence and Combustion, Vol. 84(2), pp.295-324, 2010

43. Nicoud F. and Ducros F., Subgrid-scale stress modelling based on the square of the velocity gradient, Flow Turbulence and Combustion, Vol. 62(3), pp.183-200, 1999

44. Patel N. and Menon S., Simulation of spray-turbulence-flame interactions in a lean direct injection combustor. Combust. Flame 153, 228?257, 2008

45. Poinsot T. and Lele S., Boundary conditions for direct simulations of compressible reacting flows, Journal of Computational Physics, Vol. 101(1), pp.104-129, 1992

46. Pozorski, J. and Apte, V., Filtered particle tracking in isotropic turbulence and stochastic modeling of subgrid-scale dispersion, International Journal of Multiphase Flow, Vol. 35, pp.118-128, 2009

47. Primary atomization of a liquid jet in crossflow, Physics of Fluids, Vol. 23(9), 2011

48. Reeks, M., On a kinetic equation for the transport of particles in turbulent flows, Physics of Fluids, Vol. 3, pp.446-456, 1991

49. Reeks, M., On the probability density function equations for particle dispersion in a uniform shear flow, Journal of Fluid Mechanics, Vol. 522, pp.263-302, 2005

50. Riley J.J., and Patterson G.S., Diffusion experiments with numerically integrated isotropic turbulence, Physics of Fluids, 17: 292-297, 1974 
51. Roux A., Simulation aux grandes échelles d'un statoréacteur, Ph.D. Thesis, Université de Toulouse, 2009, available on http://ethesis.inptoulouse.fr/archive/00000970/

52. Sanjosé M., Lederlin T., Gicquel L., Cuenot B., Pitsch H., Garcia-Rosa N., Lecourt R. and Poinsot T., LES of two-phase reacting flows, Center of Turbulence Research, Proceedings of The Summer Program, pp. 251-263, 2008

53. Sanjosé M., Évaluation de la méthode Euler-Euler pour la simulation numérique aux grandes échelles des chambres carburant liquide , Ph.D. Thesis, Université de Toulouse, Toulouse, 2009, available on http://ethesis.inptoulouse.fr/archive/00001026/

54. Sanjosé M., Senoner J.M., Jaegle F., Cuenot B., Moreau S., and Poinsot T., Fuel Injection Model for Euler-Euler and Euler-Lagrange Large-Eddy Simulations of an evaporating spray inside an aeronautical combustor, International Journal of Multiphase Flows, 37(5):514-529, 2011

55. Selle L., Lartigue G., Poinsot T., Koch R., Schilmacher K.-U, Krebs W., Prade B., Kaufmann P. and Veynante D., Compressible large-eddy simulation of turbulent combustion in complex geometry on unstructured meshes, Combustion and Flame, 137(4): 489-505, 2004

56. Senoner, J.M., Simulation aux grandes échelles de l'écoulement diphasique dans un bruleur aéronautique par une approche Euler-Lagrange, Ph.D. Thesis, Université de Toulouse, 2010, available on http://ethesis.inptoulouse.fr/archive/00001218/

57. Simonin, O., Février, P. and Lavieville, J., On the spatial distribution of heavy particle velocities in turbulent flow: from continuous field to particulate chaos, Journal of Turbulence, 3(1), pp. 1-40, 2002

58. Sirignano W.A., Fluid dynamics and transport of droplets and sprays, Cambridge University Press, New York, USA, 1999

59. Vié A., Martinez L., Jay S., Benkenida, A. and Cuenot B., Validation of the Eulerian mesoscopic approach in particle-charged homogeneous isotropic decaying turbulence in the scope of large eddy simulation of fuel sprays, 11th International Conference of Liquid Atomization and Spray Systems, Vail, USA, July 2009

60. Versaevel, P., Combustion laminaire diphasique: Etude théorique et expérimentale, Ph.D. Thesis, Ecole Centrale Paris, 1996

61. Vié A., Simulation aux grandes échelles d'écoulements diphasiques turbulents phase liquide dispersée , Ph.D. Thesis, Université de Toulouse, 2010, available on http://ethesis.inp-toulouse.fr/archive/00001395/

62. Vié A., Laurent F., and Massot M., Size-velocity correlations in high order moment methods for polydisperse evaporating sprays: modelling and numerical issues, Submitted to Journal of Computational Physics, 2011, available online on http://hal.archives-ouvertes.fr/hal-00626869/en

63. Von Neumann, J. and Richtmeyer, R.D., A method for the numerical calculation of hydrodynamic shocks, J. Appl. Phys., Vol. 21, pp. 232-237, 1950

64. Williams F.A., Spray Combustion and atomization, Physics of Fluids, Vol. 1, pp.541-545, 1958

65. Yuan, C. and Fox R.O., Conditional Quadrature Method of Moments for Kinetic Equations, Journal of Computational Physics, 230(22),pp. 8216-8246, 2011

66. Zaichik, L., Simonin, O. and Alipchenkov, V.M. , An Eulerian approach for large eddy simulation of particle transport in turbulent flows, Journal of Turbu- 
lence, Vol. 10 (4), pp. 1-21, 2009

67. Zaichik, L., and Alipchenkov, V.M. , Statistical models for predicting particle dispersion and preferential concentration in turbulent flows, International Journal of Heat and Fluid Flow, Vol. 26, pp. 416-430, 2005 